\title{
Existência global para equações da onda semilineares
}

Nestor Felipe Castañeda Centurión

DISSERTAÇÃO APRESENTADA

$\mathrm{AO}$

INSTITUTO DE MATEMÁTICA E ESTATÍSTICA

DA

UNIVERSIDADE DE SÃO PAULO

PARA

OBTENÇÃO DO GRAU DE MESTRE

EM

MATEMÁTICA APLICADA

Área de Concentração: Matemática Aplicada

Orientador: Prof. Dr. Pedro Paulo Serpa Schirmer

Durante a elaboração deste trabalho o autor recebeu auxílio financeiro da CNPq.

-São Paulo, fevereiro de 2003- 


\title{
Existência Global para \\ Equações da Onda \\ Semilineares
}

\author{
Este exemplar corresponde à redação \\ final da dissertação corrigida e defendida \\ por Nestor Felipe Castañeda Centurión \\ e aprovada pela comissão julgadora.
}

São Paulo, fevereiro de 2003.

Banca examinadora:

- Prof. Dr. Pedro Paulo Serpa Schirmer (Presidente) - IME-USP

- Prof. Dr. Severino Toscano do Rego Mello-IME-USP

- Prof. Dr. Oscar Fotunato V. Erazo - IME-USP 
Dedicado a mis padres Jorge y Esther.

Para mis hermanos Julia, Carmen, Carlos, Jorge y Miguel.

Por todo lo que significan para mí.

$Y$ en especial para mi hijo Cesar Levi quien siempre estuvo en mi corazón nutriendo mi alma para continuar.

Los Amo. 


\section{Agradecimentos}

Ao Povo Brasileiro representado pelo CNPq por me permitir conhecer seu país e parte da sua cultura.

Ao Prof. Dr. Pedro Paulo Schirmer por sua orientação acadêmica e seu grande apoio pessoal.

A meu amigo Jorge Julca por sua amizade e companheirismo nestes anos de percurso juntos.

Ao Walter e ao Calixto por terem feito muito mais fácil minha adaptação no Brasil, e a Ângela por me transmitir, no dia a dia do CRUSP, seu bom astral.

A Regina e a Cida por serem pessoas tão especiais, sempre irradiando alegria e carinho; e por sua imensa colaboração na revisão ortográfica.

Finalmente aos meus queridos colegas peruanos com os quais sempre compartilhei momentos agradáveis. 


\section{Resumo}

Neste trabalho estudamos o problema de existência e unicidade para as equações da onda semilineares do tipo $\square u=F_{p}(u)$, com dados de Cauchy $u(0, x)=\varepsilon f(x)$ e $u_{t}(0, x)=\varepsilon g(x)$, considerando $f, g \in C_{c}^{\infty}\left(\mathbb{R}^{n}\right), \varepsilon>0$ pequeno e $\left|(\partial / \partial u)^{j} F_{p}(u)\right| \leq$ $C_{p}|u|^{p-j}$ para $j=0,1$. O caso modelo deste problema é $\square u=|u|^{p}$, o qual estudamos separadamente em três dimensões espaciais.

Com base no trabalho de Georgiev, Lindblad e Sogge estabelecemos o domínio preciso de potências $p$, para as quais a existência de uma solução fraca global está garantida. Para isso usamos um novo tipo de estimativas de Strichartz, as quais contém pesos que envolvem potências da distancia de Lorentz. 


\section{Abstract}

In this work we study the problem of existence and unicity for the semilinear wave equations of the type $\square u=F_{p}(u)$, with Cauchy data $u(0, x)=\varepsilon f(x)$ and $u_{t}(0, x)=$ $\varepsilon g(x)$, with $f, g \in C_{c}^{\infty}\left(\mathbb{R}^{n}\right), \varepsilon>0$ a small number and $\left|(\partial / \partial u)^{j} F_{p}(u)\right| \leq C_{p}|u|^{p-j}$ for $j=0,1$. The model case of this problem is $\square u=|u|^{p}$, which is studied separately in three spacial dimensions.

Based in Georgiev, Lindblad and Sogge's work, we stablish the sharp range of powers $p$, for which the existence of a weak global solution is warranted. For this purpose we use a new kind of Strichartz's estimates, which contains weights that envolves powers of Lorentz distance. 


\section{Índice}

Agradecimentos

Resumo

Abstract

vi

Introdução

1 A semente de Fritz John 5

1.1 Blow Up em três dimensões espaciais. . . . . . . . . . . 5

1.2 Existência global em três dimensões espaciais . . . . . . . . . 12

2 Estimativas de Strichartz 18

2.1 Convoluções com Núcleos que possuem suas singularidades sobre a esfera . . . . . . . . . . . . . . . . . . 18

2.2 Estimativa a priori para a equação da onda . . . . . . . . . 24

3 Existência Global em $n$ Dimensões Espaciais $\quad 27$

3.1 Formulação do Problema e o Teorema Central . . . . . . . . 27

3.2 Processo Iterativo . . . . . . . . . . . . . . . . . . . . . . . . . . 29

3.3 Demonstração do Teorema Central . . . . . . . . . . . . . . 33 
4 Estimativas de Strichartz com Pesos 36

4.1 Roteiro da Demonstração . . . . . . . . . . . . . . 36

4.2 Contribuição de $F$ para tempos relativamente grandes . . . . 37

A Teoremas de Interpolação $\quad 42$

A.1 Teorema de Interpolação de M. Riesz . . . . . . . . . . . . . . . 42

A.2 Teorema de Interpolação de Marcinkiewicz . . . . . . . . . . 43

A.3 Teorema de Interpolação de Stein . . . . . . . . . . . . . . . 44

B Teorema de Extensão de Calderón $\quad 47$

B.1 Espaços de Sobolev de Ordem Inteira . . . . . . . . . . . . 47

B.2 O Teorema de Extensão de Calderón . . . . . . . . . . . . 48

C Desigualdades e Teoremas Mencionados $\quad 50$

C.1 Desigualdade de Hölder . . . . . . . . . . . . . . . . 50

C.2 Desigualdade de Hardy-Littlewood-Sobolev . . . . . . . . . 50

C.3 Teorema de Plancherel . . . . . . . . . . . . . . . 51

Referências Bibliográficas $\quad 53$ 


\section{Introdução}

O objetivo desta dissertação consiste em estudar o problema de existência global de soluções, em $n$ dimensões espaciais, para a equação da onda semilinear do tipo

$$
\left\{\begin{array}{l}
\square u=F_{p}(u), \quad(t, x) \in \mathbb{R}_{+}^{1+n} \\
u(0, x)=\varepsilon f(x), \quad \partial_{t} u(0, x)=\varepsilon g(x),
\end{array}\right.
$$

considerando $p>1, f, g \in C_{c}^{\infty}\left(\mathbb{R}^{n}\right), \varepsilon>0$ suficientemente pequeno e $F_{p}$ um termo não linear, tal que

$$
\left|\left(\frac{\partial}{\partial u}\right)^{j} F_{p}(u)\right| \leq C_{j}|u|^{p-j}, \quad j=0,1 .
$$

Em outras palavras, a partir de $n$ dado e tendo-se controle através de $\varepsilon$ sobre os suportes dos dados iniciais, busca-se saber o domínio preciso de valores de $p$, para os quais este problema possui uma solução fraca global.

A condição imposta sobre $F_{p}$ tem sua origem no caso modelo $F_{p}=|u|^{p}$. É justamente nesse caso que Fritz John, no seu trabalho pioneiro (ver [9]), demonstrou que para $n=3$ existem sempre soluções globais do problema, se considerarmos $p>1+\sqrt{2}$ e $\varepsilon$ suficientemente pequeno. Ele mostrou também que esse valor de $p$ é crítico, no sentido que para $p<1+\sqrt{2}$, as soluções explodem em tempo finito, ou seja, quando isso ocorre tem lugar o fenômeno conhecido como Blow-up. O que acontecia no caso $p=1+\sqrt{2}$ ? Foi a pergunta que surgiu, então. A resposta foi dada tempo depois por Schaeffer (ver [14]), o qual demonstrou que o Blow-up pode se apresentar para $\varepsilon$ arbitrariamente pequeno. Assim, o caso $n=3$ resolveu-se de forma satisfatória. 
O número $1+\sqrt{2}$ aparece, pela primeira vez, no trabalho de Strauss sobre teoria de espalhamento, para equações de Schrödinger semilineares com amplitude pequena (ver [19]). Baseado nisso, ele fez sua famosa e altamente intuitiva conjetura (ver [20]):

Para $n \geq 2$ o problema (1) possui sempre soluções globais se $\varepsilon>0$ for suficientemente pequeno e $p$ for maior que uma potência crítica, solução da seguinte equação quadrática:

$$
(n-1) p_{c}^{2}-(n+1) p_{c}-2=0 \quad, \quad p_{c}>1
$$

Notemos que ao considerarmos $n=3$ obtemos como solução de (2) a potência crítica $p_{c}=1+\sqrt{2}$, ou seja, a conjetura tinha a base da sua validade no caso $n=3$.

Depois de colocada, a conjetura foi rapidamente verificada por Glassey, no caso $n=2$ (ver [5]). Um resultado muito mais relevante foi estabelecido por Sideris, que estendeu os resultados sobre Blow-up de F. John, mostrando que, para todo $n$ este fenômeno pode-se apressentar considerando dados iniciais arbitrariamente pequenos, sempre que $p<p_{c}$ (ver [15]). Assim, a "metade" da conjetura estava resolvida.

Em uma primeira tentativa por resolver a outra "metade" do problema, tentou-se achar uma estimativa análoga à estabelecida nessa parte por John. Muito cedo esse procedimento falhou, pois a passagem principal de John baseia-se em uma estimativa equivalente à seguinte:

$$
\begin{gathered}
\left|t(t-|x|)^{p-2} w\right|_{L^{\infty}\left(\mathbb{R}_{+}^{1+3}\right)} \leq C_{p}\left|t^{p}(t-|x|)^{p(p-2)} F\right|_{L^{\infty}\left(\mathbb{P}_{+}^{1+3}\right)}, \\
\text { se } F(t, x)=0, t-|x| \leq 1, \text { e } 1+\sqrt{2}<p \leq 3,
\end{gathered}
$$

onde $w$ é solução para $n=3$ do problema

$$
\left\{\begin{array}{l}
\square w(t, x)=F(t, x) \quad, \quad(t, x) \in \mathbb{R}_{+}^{1+n} \\
0=w(0, \cdot)=\partial_{t}(0, \cdot),
\end{array}\right.
$$

e, infelizmente, essa estimativa pontual não pode ser estabelecida em dimensões maiores, devido ao fato de que as soluções fundamentais do operador de D'Alembert não são mais medidas quando $n \geq 4$. 
Passou-se quase uma década para que a parte de existência global fosse abordada com sucesso para $n>3$. Os primeiros trabalhos sempre obtiveram resultados positivos, mas para casos particulares. Por exemplo, Zhou mostrou que quando $n=4$, valor para o qual $p_{c}=2$, sempre existem soluções globais de (1), considerando dados pequenos e $p>p_{c}$, (ver [23]). Lindblad e Sogge também demonstraram a parte de existência global agora no caso $n \leq 8$ (ver [12]). Nesse mesmo trabalho foram um pouco mais longe, pois demonstraram que sob as hipóteses de simetria esférica a conjetura de Strauss era válida para qualquer $n \geq 3$. Salientamos que tal resultado foi obtido também por Kubo para n ímpar (ver [10]). Outro resultado interessante alcançado por Linblad e Sogge foi a obtenção de uma potência conformemente invariante, $p_{\text {conf }}=(n+3) /(n-1)$, demonstrando que a existência global sempre está garantida para $p>p_{\text {conf }}($ ver $[11])$.

Novas idéias foram aparecendo. Foi assim, que Georgiev inicia, em seu trabalho [3], a idéia de utilizar, na demonstração da existência global, certa versão com pesos das já conhecidas estimativas de Strichartz para o problema (3) (ver [22]), isto é, uma versão com pesos de

$$
\|w\|_{L^{2(n+1) /(n-1)\left(\mathbb{R}_{+}^{1+n}\right)}} \leq C\|F\|_{L^{2(n+1) /(n+2)\left(\mathbb{R}_{+}^{1+n}\right)}} .
$$

A demonstração cabal da conjetura achava-se próxima e, assim, um ano depois, em um trabalho conjunto de Georgiev, Lindblad e Sogge, (ver [4]), foi provada a existência de uma solução fraca global $u$, do problema (1), com $n \geq 3, p_{c}<p<p_{\text {conf }}$, $F_{p}$ fixo e $\varepsilon$ suficientemente pequeno, a qual satisfaz

$$
\left(1+\left.\left|t^{2}-\right| x\right|^{2} \mid\right)^{\gamma} u \in L^{p+1}\left(\mathbb{R}_{+}^{1+n}\right)
$$

para algum $\gamma$, tal que

$$
\frac{1}{p(p+1)}<\gamma<\frac{(n-1) p-(n+1)}{2(p+1)} .
$$

A prova deste fato está baseada, fundamentalmente, na seguinte estimativa para a solução do problema (3), considerando $n \geq 2$ :

$$
\left\|\left(t^{2}-|x|^{2}\right)^{\gamma_{1}} w\right\|_{L^{q\left(\mathbb{R}_{+}^{1+n}\right)}} \leq C_{q, \gamma}\left\|\left(t^{2}-|x|^{2}\right)^{\gamma_{2}} F\right\|_{L^{q /(q-1)}\left(\mathbb{R}_{+}^{1+n}\right)},
$$


sempre que $2 \leq q \leq \frac{2(n+1)}{n-1}$

$$
\gamma_{1}<n\left(\frac{1}{2}-\frac{1}{q}\right)-\frac{1}{2} \quad \text { e } \quad \gamma_{2}>\frac{1}{q}
$$

e $F(t, x)=0$ se $t-|x| \leq 1$.

Agora, passamos a descrever sucintamente a estrutura da dissertação, no primeiro capítulo será estudado o trabalho precursor de Fritz John, tomando-se por referência. seu próprio trabalho [9].

O segundo capítulo é dedicado ao estudo das estimativas de Strichartz para operadores que possuem suas singularidades sobre a esfera, e a aplição desses resultados à equação da onda. As principais referências são os livros de Bergh e Löfström [1] e Gel'fand e Silov [2]; e os trabalhos de Hörmander [7], Littman [13], Stein [18] e Strichartz [21, 22].

No capítulo seguinte será estudada a demonstração de (4) a partir de (5) e (6). As principais referências são o livro de Hörmander [8]; e os trabalhos de Georgiev, Lindblad e Sogge [4], Lindblad e Sogge [11, 12] e Strichartz [21, 22].

O quarto e último capítulo versa sobre a demontração de (5), levando-se em consideração (6). São referências os livros de Bergh e Löfström [1] e Sogge [17]; e os trabalhos de Georgiev [3], Georgiev, Lindblad e Sogge [4], Lindblad e Sogge $[11,12]$, Elias Stein [18], Hörmander [6], Sogge e Stein [16] e Strichartz [21, 22]. 


\section{A semente de Fritz John}

Neste capítulo estudaremos o trabalho precursor de Fritz John feito sobre o problema. de Cauchy (ver [9])

$$
\left\{\begin{array}{l}
\square u=|u|^{p} \\
u(0, x)=f(x) \quad, \quad u_{t}(0, x)=g(x) \\
f \in C^{3}\left(\mathbb{R}^{3}\right) \quad \text { e } \quad g \in C^{2}\left(\mathbb{R}^{3}\right) .
\end{array}\right.
$$

Veremos como nesse problema a potência $p=1+\sqrt{2}$ é crítica, no sentido que para. valores maiores que $1+\sqrt{2}$ sempre existe uma solução global do problema, e para valores menores que essa potência a solução explode em tempo finito.

\subsection{Blow Up em três dimensões espaciais.}

Seja $\square$ o operador de D'Alembert em três dimensões espaciais, agindo sobre funções do tipo $u(t, x)$, onde $t \geq 0$ e $x \in \mathbb{R}^{3}$. Abreviando-se este último escreveremos $(t, x) \in$ $\mathbb{R}_{+}^{1+3}$.

Definição 1.1 Para $\left(t_{0}, x^{0}\right) \in \mathbb{R}_{+}^{1+3}$, definimos os cones característicos sólidos do futuro e do passado com vértice em $\left(t_{0}, x^{0}\right)$ :

$$
\begin{gathered}
\Gamma^{+}\left(t_{0}, x^{0}\right)=\left\{(t, x):\left|x-x^{0}\right| \leq t-t_{0}, t \geq 0\right\} \\
\Gamma^{-}\left(t_{0}, x^{0}\right)=\left\{(t, x):\left|x-x^{0}\right| \leq t_{0}-t, t \geq 0\right\} .
\end{gathered}
$$


Teorema 1.2 Seja $p$ uma constante satisfazendo $1<p<1+\sqrt{2}$ e seja u uma solução global do problema (1.1).

Além disso, para certo ponto $\left(t_{0}, x^{0}\right) \in \mathbb{R}_{+}^{1+3}$

$$
u^{0} \geq 0, \quad(t, x) \in \Gamma^{+}\left(t_{0}, x^{0}\right),
$$

sendo $u^{0}$ a solução da equação linear homogênea $\square u^{0}=0$ com os mesmos dados iniciais $f$ e $g$.

Então, u tem suporte compacto e $\operatorname{supp}(u) \subset \Gamma^{-}\left(t_{0}, x^{0}\right)$.

Prova. Faremos uso da expressão explícita da solução global em três dimensões espaciais do problema linear $\square u=w(t, x)$, para $(t, x) \in \mathbb{R}_{+}^{1+3}$ com os mesmos dados iniciais do problema $(1.1)$ e $w \in C^{2}\left(\mathbb{R}_{+}^{1+3}\right)$. Isto é, $u=u^{0}+L w$, onde

$$
\begin{gathered}
u^{0}(t, x)=\frac{t}{4 \pi} \int_{|\xi|=1} g(x+t \xi) d \omega_{\xi}+\frac{\partial}{\partial t}\left\{\frac{t}{4 \pi} \int_{|\xi|=1} f(x+t \xi) d \omega_{\xi}\right\} \quad \mathrm{e} \\
L w(t, x)=\frac{1}{4 \pi} \int_{0}^{t}(t-s) d s \int_{|\eta|=1} w(s, x+(t-s) \eta) d \omega_{\eta} .
\end{gathered}
$$

Definindo a média esférica de uma função $w(t, x)$, sobre a esfera de raio $r$ em um ponto $x^{0}$, como

$$
\widetilde{w}(t, r)=\frac{1}{4 \pi} \int_{|\xi|=1} w\left(t, x^{0}+r \xi\right) d \omega_{\xi},
$$

podemos calcular a média esférica do operador $L w$

$$
\widetilde{L w}(t, r)=\int_{0}^{t} d s \int_{|r-t+s|}^{r+t-s} \frac{\lambda}{2 r} \widetilde{w}(s, \lambda) d \lambda .
$$

Expressaremos essa identidade por $\widetilde{L w}=P \widetilde{w}$, onde o operador $P$, agindo sobre funções $\sigma(t, r)$ com domínio $r, t \geq 0$, é definido

$$
P \sigma(t, r)=\iint_{R_{r, t}} \frac{\lambda}{2 r} \sigma(s, \lambda) d \lambda d s,
$$

onde $R_{r, t}$ denota o conjunto

$$
R_{r, t}=\{(s, \lambda): t-r<s+\lambda<t+r, s-\lambda<t-r, 0<s\} \quad \text { no plano } \lambda s .
$$




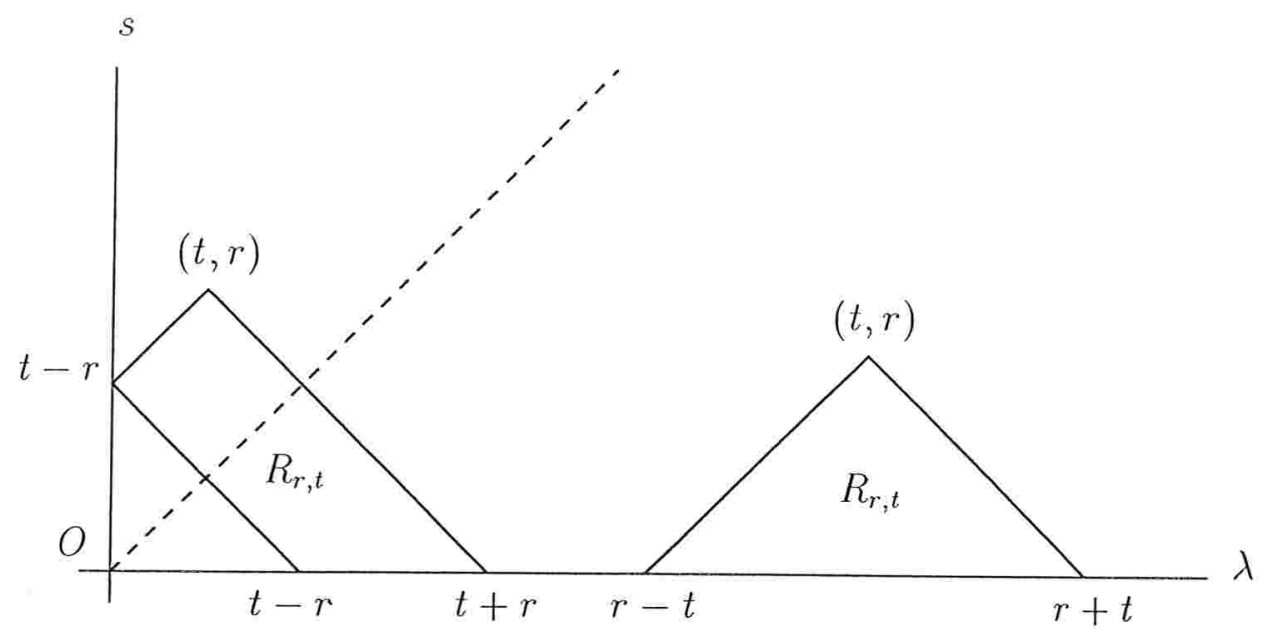

Figura 1.1: Esboço do conjunto $R_{r, t}$ no plano $\lambda s$.

Observemos que $\sigma \geq 0$ implica $P \sigma \geq 0$, pois $\lambda \geq 0$ em $R_{r, t}$. Assumamos que $u(t, x)$ é uma solução global de (1.1), e que o correspondente $u^{0}$ satisfaz (1.2) para $\operatorname{algum}\left(t_{0}, x^{0}\right)$. Logo,

$$
u \geq L|u|^{p}, \quad(t, x) \in \Gamma^{+}\left(t_{0}, x^{0}\right) .
$$

Demonstraremos nossa afirmação por contradição. Assim, negando a nossa tese, obtemos a existência de um ponto $\left(t_{1}, x^{1}\right) \in \Gamma^{+}\left(t_{0}, x^{0}\right)$, onde $u$ não se anula, isto é, $u\left(t_{1}, x^{1}\right) \neq 0$. Ao chamarmos $t_{2}=t_{1}+\left|x^{1}-x^{0}\right|$, decorre que $\left(t_{2}, x^{0}\right) \in \Gamma^{+}\left(t_{0}, x^{0}\right)$; isso associado a (1.7) nos permite concluir que $u\left(t_{2}, x^{0}\right)>0$, pois a nossa $u$ é contínua e não se anula em $\left(t_{1}, x^{1}\right)$, que pertence ao domínio de integração do operador $L$, o qual, agindo sobre $|u|^{p}$, é, obviamente, não negativo.

Como $\Gamma^{+}\left(t_{2}, x^{0}\right) \subset \Gamma^{+}\left(t_{0}, x^{0}\right),(1.7)$ é válida para $\left|x-x^{0}\right|<t-t_{2}$. Por outro lado, $|u|^{p}$ para $p \geq 1$ é uma função convexa em relação a $u$; assim, $\widetilde{|u|^{p}} \geq|\widetilde{u}|^{p}$. Da união dessas observações com a identidade obtida em (1.5), para $0 \leq r \leq t-t_{2}$, temos

$$
\widetilde{u}(t, r) \geq \widetilde{L|u|^{p}}(t, r)=P \widetilde{|u|^{p}}(t, r) \geq P|\widetilde{u}(t, r)|^{p}=\iint_{R_{r, t}} \frac{\lambda}{2 r}|\widetilde{u}(s, \lambda)|^{p} d \lambda d s .
$$

Ademais, considerando $u\left(t_{2}, x^{0}\right)>0$ e a definição de média esférica, temos

$$
\widetilde{u}\left(t_{2}, 0\right)>0 .
$$


Veremos que (1.8) agregado a (1.9) implicam blow-up da $u$. Isso ocorrerá obtendo-se sucessivas cotas inferiores da $\tilde{u}$ cada vez maiores.

Por meio de (1.9) podemos achar um $\delta>0$ suficientemente pequeno, tal que $\widetilde{u}\left(t_{2}+\right.$ $\delta / 2, \delta / 2)>0$. A seguir, definimos as regiões

$$
T=\left\{(s, \lambda): t_{2}+\delta \leq s+\lambda \leq t_{2}+2 \delta, s-\lambda \leq t_{2}\right\}
$$

$\mathrm{e}$

$$
S=\left\{(s, \lambda): t_{2}+2 \delta \leq s+\lambda, t_{2} \leq s-\lambda \leq t_{2}+\delta\right\}
$$

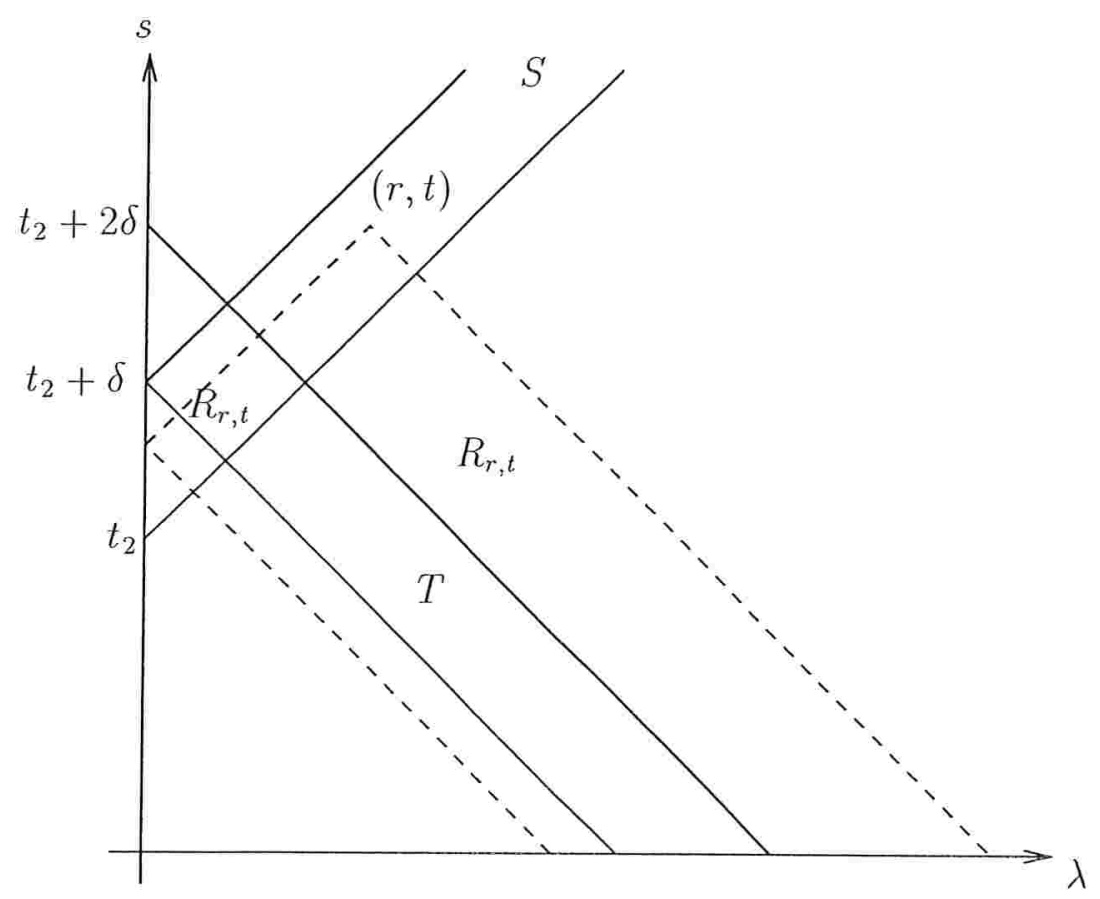

Figura 1.2: Esboços dos conjuntos $R_{r, t}, T$ e $S$ no plano $\lambda s$.

Notemos que para $(t, r) \in S, T \subset R_{r, t}$ e $0 \leq r \leq t-t_{2}$. Isso associado a (1.8) nos leva a concluir que para $(t, r) \in S$

$$
\widetilde{u}(t, r) \geq \iint_{T} \frac{\lambda}{2 r}|\widetilde{u}(s, \lambda)|^{p} d \lambda d s=\frac{C}{r},
$$

onde $C$ é uma constante positiva, pois $\left(t_{2}+\delta / 2, \delta / 2\right) \in T$ e $\widetilde{u}\left(t_{2}+\delta / 2, \delta / 2\right)>0$. Definamos o conjunto $\Sigma=\left\{(t, r) / \quad 0 \leq r \leq t-t_{2}-2 \delta\right\}$ e para qualquer $(t, r) \in \Sigma$, 
definem-se os conjuntos:

$$
S_{r, t}=\left\{(s, \lambda): t-r<s+\lambda<t+r, t_{2}<s-\lambda<t_{2}+\delta\right\}
$$

e

$$
T_{r, t}=\left\{(s, \lambda): t-r<s+\lambda<t+r, t_{2}+2 \delta<s-\lambda<t-r\right\} .
$$

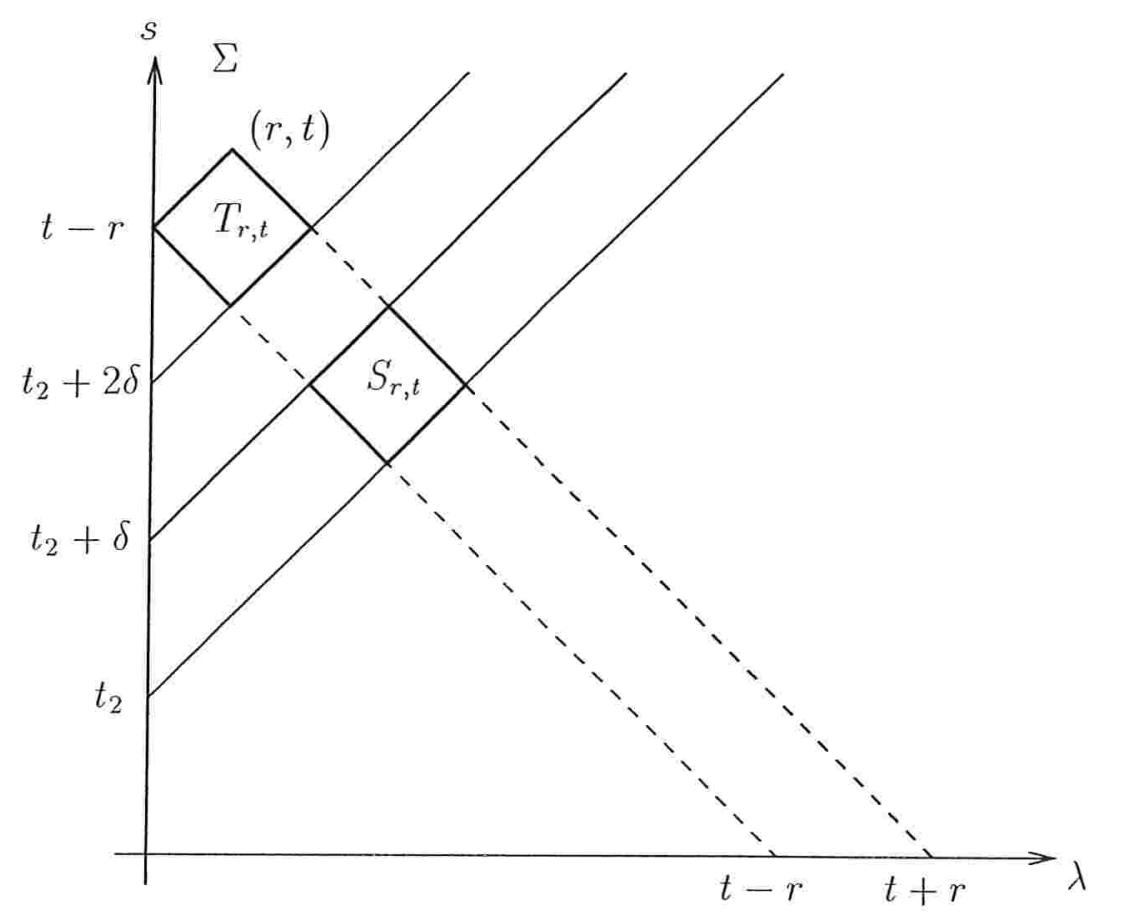

Figura 1.3: Esboço dos conjuntos $S_{r, t}$ e $T_{r, t}$ no plano $\lambda s$.

Decorre dessa definição que para $(t, r) \in \Sigma$, valem $S_{r, t} \subset R_{r, t}, T_{r, t} \subset R_{r, t}, S_{r, t} \subset S$ e $T_{r, t} \subset \Sigma$. Essas afirmações agregadas a (1.8) e (1.10) implicam

$$
\widetilde{u}(t, r) \geq \frac{C^{p}}{2 r} \iint_{S_{r, t}} \lambda^{1-p} d \lambda d s, \quad(t, r) \in \Sigma .
$$

Fazendo a mudança de variáveis $\alpha=s+\lambda, \beta=s-\lambda$ e levando-se em conta que para $p>1$ a função $(\alpha-\beta)^{1-p}$ é decrescente em $\alpha$ e crescente em $\beta$, obtemos

$$
\widetilde{u}(t, r) \geq \delta C^{p} 2^{p-2}\left(t+r-t_{2}\right)^{1-p}, \quad(t, r) \in \Sigma .
$$


Da própria definição de $\Sigma$ segue que $\delta \leq t-r-t_{2} \leq t+r-t_{2} \leq t+r$ para qualquer $(t, r) \in \Sigma$. Essa desigualdade permite estimar por baixo $\left(t+r-t_{2}\right)^{1-p}$ para qualquer $p>1$. Logo, de (1.11) chegamos a

$$
\widetilde{u}(t, r) \geq C_{0}(r+t)^{-q_{0}}, \quad(t, r) \in \Sigma,
$$

onde $C_{0}$ e $q_{0}$ são constantes positivas que não dependem de $(t, r)$, e em particular

$$
1 \leq q_{0}= \begin{cases}1, & 1<p \leq 2 \\ p-1, & 2<p<1+\sqrt{2}\end{cases}
$$

Veja que neste momento a inclusão do limite superior $1+\sqrt{2}$ é artificial. De fato, (1.12) é válida se $q_{0}=p-1$ para todo $p>2$.

Admitamos de modo geral a possibilidade de estabelecer uma estimativa da forma

$$
\widetilde{u}(t, r) \geq C(t+r)^{-q}(t+r-\tau)^{a}(t+r)^{-b}, \quad(t, r) \in \Sigma,
$$

onde $\tau=t_{2}+2 \delta, C>0, q \geq 1, a \geq 0$ e $b \geq 0$. Então, por (1.8) e já que $T_{r, t} \subset R_{r, t}$, obtemos uma integral sobre $T_{r, t}$ como cota inferior de $\widetilde{u}(t, r)$; nessa integral fazemos a mudança de variáveis, anteriormente mencionada, para obter

$$
\widetilde{u}(t, r) \geq \frac{C^{p}(t-r-\tau)^{p a+2}}{4(p a+1)(p a+2)(t-r)^{p b}} I(t, r), \quad(t, r) \in \Sigma,
$$

onde $I(t, r)=\int_{t-r}^{t+r} \alpha^{-p q} d \alpha$.

Estimando por baixo $I(t, r)$, chegamos a

$$
I(t, r) \geq \begin{cases}2^{1-p q}(t+r)^{-1}(t-r)^{1-p q}, & \frac{1}{2}(t+r)<t-r \\ \frac{1-2^{1-p q}}{2(p q-1)}(t+r)^{-1}(t-r)^{1-p q}, & 0<t-r<\frac{1}{2}(t+r)\end{cases}
$$

Juntando nossas duas últimas conclusões, chegamos a uma estimativa do tipo

$$
\widetilde{u}(t, r) \geq C^{*}(t+r)^{-1}(t-r-\tau)^{a^{*}}(t-r)^{-b^{*}}, \quad(t, r) \in \Sigma
$$

onde $a^{*}=p a+2, b^{*}=p b+q p-1, C^{*}=C^{p}(p a+2)^{-2} D_{q} ; D_{q}$ é a quarta parte do mínimo dos coeficientes das cotas inferiores em (1.15). Usando os valores $a=b=0$, $q=q_{0}$ e $C=C_{0}$, dados em (1.12), vemos que (1.16) é válida desde que $a^{*}=2$, 
$b^{*}=q_{0} p-1$ e $C^{*}=C_{0}^{p} D_{q_{0}} / 4$.

Definimos em geral as seqüências $a_{k}, b_{k}$ e $c_{k}$ para $k=1,2,3, \ldots$ da seguinte forma:

$$
\left\{\begin{array}{l}
a_{1}=a^{*}, \quad b_{1}=b^{*}, \quad C_{1}=C^{*} \\
a_{k+1}=p a_{k}+2, \quad b_{k+1}=p b_{k}+p-1, \quad C_{k+1}=\left(p a_{k}+2\right)^{-2} D_{1} C_{k}^{p} .
\end{array}\right.
$$

Logo, (1.14) será verdadeira com $C=C_{k}, a=a_{k}, b=b_{k}, q=1$ para $k \geq 1$. Resolvendo (1.17), obtemos que $a_{k}=2\left(p^{k}-1\right) /(p-1), b_{k}=q_{0} p^{k}-1 \mathrm{e}$, assim,

$$
C_{k+1}=\left(a_{k+1}\right)^{-2} D_{1} C_{k}^{p} \geq \frac{1}{4(k+1)^{2} p^{2 k}} D_{1} C_{k}^{p}
$$

Utilizando essa estimativa reiteradas vezes até chegar a $C_{1}=C^{*}$ e usando o fato que $e^{\log x}=x$, obtemos

$$
C_{k} \geq \exp \left[p^{k}\left(\frac{1}{p} \log C^{*}-\sum_{j=1}^{k-1} \frac{2 \log (j+1)+2 j \log (p)-\log \left(D_{1} / 4\right)}{p^{j+1}}\right)\right] .
$$

Segue dessa expressão que para $k$, suficientemente grande, $C_{k} \geq \exp \left(E p^{k}\right)$, onde

$$
E=\frac{1}{p} \log C^{*}-\sum_{j=1}^{\infty} \frac{2 \log (j+1)+2 j \log (p)-\log \left(D_{1} / 4\right)}{p^{j+1}} .
$$

Substituindo em (1.14) $C, a, b$ e $q$, por $C_{k}, a_{k}, b_{k}$ e 1, respectivamente obtemos

$$
\tilde{u}(t, r) \geq \frac{t-r}{(t+r)(t-r-\tau)^{2 /(p-1)}} \exp \left[p^{k} J(t, r)\right] \quad, \quad(t, r) \in \Sigma .
$$

Aqui,

$$
J(t, r)=E+\frac{2}{p-1} \log (t-r-\tau)-q_{0} \log (t-r) .
$$

Dessa desigualdade advem a importância de definir $q_{0}$ como em (1.13), pois é necessário que $2 /(p-1)>q_{0}$. Perceba também que é neste ponto que aparece a cota superior $1+\sqrt{2}$. Finalmente vejamos que $J(t, r) \rightarrow \infty$ quando $t-r \rightarrow \infty$. Assim, por (1.18) concluímos que quando $k \rightarrow \infty, \widetilde{u}(t, r) \rightarrow \infty$ para todo $r, t$, tal que $t-r$ seja suficientemente grande. Portanto, $u$ não é uma solução global e este fato em si é uma contradição. 


\subsection{Existência global em três dimensões espaciais}

Teorema 1.3 O problema (1.1) possui soluções globais para quaisquer dados iniciais $f$ eg suficientemente regulares, com suporte na bola de raio $\rho$, sempre que $p>1+\sqrt{2}$, $\left|D^{\alpha} f\right|$ para $|\alpha| \leq 2$ e $\left|D^{\beta} f\right|$ para $|\beta| \leq 1$ não excedam certo número positivo $\delta$, que somente depende de $\rho$.

Prova. Admitamos que $f \in C_{0}^{5}\left(\mathbb{R}^{3}\right)$ e $g \in C_{0}^{4}\left(\mathbb{R}^{3}\right)$, e mais precisamente que para certos $x^{0}$ e $\rho: f(x)=g(x)=0$ para $\left|x-x^{0}\right|>\rho$.

Sob essas hipóteses, $u^{0}$ e suas derivadas de ordem $\leq 3$ decaem, tal como $1 / t$ para $t$ grande. Para esclarecer, definamos:

$$
N_{\nu}=\sup _{\substack{|\alpha| \leq 1 \\|\beta| \leq 2 \\ x \in \mathbb{R}^{3}}}\left\{\rho^{|\alpha|}\left|D^{\alpha} f(x)\right|, \rho^{|\beta|+1}\left|D^{\beta} g(x)\right|\right\}
$$

Logo, existe uma constante universal $\gamma$, tal que ${ }^{1}$

$$
\rho^{|\alpha|}\left|D^{\alpha} u^{0}(t, x)\right|<\gamma \frac{\rho}{\rho+t} N_{\nu}, \quad(t, x) \in \mathbb{R}_{+}^{1+3} \quad \text { e } \quad|\alpha| \leq \nu,
$$

quando $\nu=0,1,2,3$.

Mostraremos a existência de uma solução global $u$ de (1.1), com dados iniciais $f$ e $g$, impondo-se que $N_{0}$, para $p$ e $\rho$ dados, seja suficientemente pequeno. Para tanto, basta encontrar uma $u(t, x)$ para a qual as expressões $D^{\alpha} u(t, x)$ com $|\alpha| \leq 2$ existam e sejam contínuas em $(t, x) \in \mathbb{R}_{+}^{1+3}$ e $u=u^{0}+L|u|^{p}$.

Lema 1.4 Seja $w(t, x)$ definida para $(t, x) \in \mathbb{R}_{+}^{1+3}$, tal que $D^{\alpha} w(t, x)$ para $|\alpha| \leq 2$ existem e são contínuas nesse domínio ${ }^{2}$. Então, $L w(t, x)$ como função de $x$ e $t$ é de classe $C^{2}$ em $\mathbb{R}_{+}^{1+3}$, tem dados iniciais nulos e satisfaz $\square u=w$.

Prova. Basta introduzir

$$
M(r, t, x)=\frac{1}{4 \pi} \int_{|\eta|=1} w(x+r \eta, t) d \omega_{\eta}, \quad r \geq 0,(t, x) \in \mathbb{R}_{+}^{1+3} .
$$

\footnotetext{
${ }^{1} \mathrm{O}$ símbolo $D^{\alpha}$ será usado para referir à diferenciação espacial $D_{x_{1}}^{\alpha_{1}} D_{x_{2}}^{\alpha_{2}} D_{x_{3}}^{\alpha_{3}}$

${ }^{2}$ Observe que não é assumida a existência das derivadas temporais de $w$.
} 
Notemos que $M(r, t, x)$ é de classe $C^{2}$ em $x, r$, uniformemente em $t$. Então, as nossas afirmações decorrem da identidade de Darboux em três dimensões espaciais: $M_{r r}(r, t, x)=\triangle_{x} M(r, t, x)$, e que através de (1.3) tem-se $L w(t, x)=\int_{0}^{t} M(r, t, x)$. Associamos a uma função contínua $w(t, x),(t, x) \in \mathbb{R}_{+}^{1+3}$ a função

$$
\bar{w}(r, t)=\sup _{\substack{\left|x-x^{0}\right|=r \\ x \in \mathbb{R}^{3}}}|w(x, t)|
$$

definida para $r, t \geq 0$.

Lema 1.5 Para $\left|x-x^{0}\right|=r, t \geq 0$

$$
|L w(t, x)| \leq \int_{0}^{t} d s \int_{|r-t+s|}^{r+t-s} \frac{\lambda}{2 r} \bar{w}(\lambda, s) d \lambda=\iint_{R_{r, t}} \frac{\lambda}{2 r} \bar{w}(\lambda, s) d \lambda d s,
$$

ou $\overline{L w} \leq P \bar{w}$, onde $P$ é o operador definido em (1.6).

Prova. Introduzindo $v(t, x)=\bar{w}\left(\left|x-x^{0}\right|, t\right)$, é fácil demonstrar que $\overline{L w} \leq \widetilde{L v}=P \widetilde{v}=$ $P \bar{v}=P \bar{w}$, onde $\widetilde{v}$ foi definida em (1.4).

Provaremos a existência de uma solução global de (1.1) mediante um processo iterativo; para tanto, introduziremos uma norma apropriada. Assim, definimos para funções $u(t, x)$, contínuas em $\mathbb{R}_{+}^{1+3}$ e com suporte em $\Gamma^{+}\left(-\rho, x^{0}\right)$, a seguinte norma:

$$
\begin{aligned}
\|u\| & =\sup _{\substack{r \geq 0 \\
t \geq 0}} \rho^{1-p}(t+r+2 \rho)(t-r+2 \rho)^{p-2} \bar{u}(r, t) \\
& =\sup _{\substack{t \geq 0 \\
x \in \mathbb{R}^{3}}} \rho^{1-p}\left(t+\left|x-x^{0}\right|+2 \rho\right)\left(t-\left|x-x^{0}\right|+2 \rho\right)^{p-2}|u(t, x)| .
\end{aligned}
$$

Dessa definição tem-se que $D^{\alpha} u^{0}$ para $|\alpha|<3$ tem norma finita, pois pelo Princípio Forte de Huygens podemos afirmar que $u^{0}(t, x)=0$ a menos que $t-\rho<\left|x-x^{0}\right|<t+\rho$. Logo, $\rho^{1-p}\left(t+\left|x-x^{0}\right|+2 \rho\right)\left(t-\left|x-x^{0}\right|+2 \rho\right)^{p-2} \leq \rho^{1-p}(2 t+3 \rho)(3 \rho)^{p-2} \leq 3^{p-1}(t+\rho) \rho^{-1}$, no suporte de $u^{0}$; assim, aplicando (1.20), chegamos a

$$
\left\|D^{\alpha} u^{0}\right\| \leq \gamma 3^{p-1} \rho^{-\nu}, \quad|\alpha|=\nu \leq 3
$$

O lema seguinte será fundamental na prova de existência.

Lema 1.6 Existe uma constante $C=C(p)$, tal que

$$
\left\|L|u|^{p}\right\| \leq C \rho^{2}\|u\|^{p}
$$


sendo $u(t, x)$ contínua em $\mathbb{R}_{+}^{1+3}$ e satisfazendo

$$
u(t, x)=0, \quad\left|x-x^{0}\right|>t+\rho .
$$

Prova. O caso é relevante quando a norma de $u$ é finita. Aplicando o lema 1.6 a $L|u|^{p}$, vemos que basta mostrar

$$
\int_{0}^{t} d s \int_{|r-t+s|}^{r+t-s} \frac{\lambda}{2 r} \bar{u}^{p}(\lambda, s) d \lambda \leq \frac{C c^{p} \rho^{p+1}}{(t+r+2 \rho)(t-r+2 \rho)^{p-2}},
$$

onde $C$ depende unicamente de $p$, tendo como hipótese que

$$
0 \leq \bar{u}(\lambda, s) \leq \frac{c \rho^{p-1}}{(s+\lambda+2 \rho)(s-\lambda+2 \rho)^{p-2}} .
$$

Além disso, por (1.24)

$$
\bar{u}(\lambda, s)=0, \quad \lambda>s+\rho .
$$

Em primeiro lugar demonstraremos (1.25), no caso $t-\rho<r<t+\rho$.

Nesse sentido, para $(\lambda, s)$ na região de integração onde $\bar{u}(\lambda, s) \neq 0$, teremos também que $s-\rho<\lambda<s+\rho$. Segue de (1.26) que $\bar{u}(\lambda, s) \leq c \rho /(2 s+\rho)$ e, portanto,

$$
\int_{0}^{t} d s \int_{|r-t+s|}^{r+t-s} \frac{\lambda}{2 r} \bar{u}^{p}(\lambda, s) d \lambda \leq c^{p} \rho^{p} \int_{0}^{t}(2 s+\rho)^{-p} k(s) d s, \quad k(s)=\frac{1}{2 r} \int_{I} \lambda d \lambda,
$$

onde $I$ é a interseção dos intervalos $[|r-t+s|, r+t-s]$ e $[|r-t+s|, s+\rho]$.

Separando os casos $r>2 \rho$ e $0<r<2 \rho$, chegamos sempre ao resultado $k(s) \leq$ $\frac{12 p(s+\rho)}{t+\rho}$. Portanto,

$$
\begin{aligned}
& \int_{0}^{t} d s \int_{|r-t+s|}^{r+t-s} \frac{\lambda}{2 r} \bar{u}^{p}(\lambda, s) d \lambda \leq \frac{12 c^{p} \rho^{p+1}}{t+\rho} \int_{0}^{t}(s+\rho)(2 s+\rho)^{-p} d s \\
\leq & \frac{12 c^{p} \rho^{3}}{t+\rho} \int_{0}^{\infty}(\mu+1)^{1-p} d \mu \leq \frac{36.3^{p-2} c^{p} \rho^{p+1}}{(p-2)(t+r+2 \rho)(t-r+2 \rho)^{p-2}} .
\end{aligned}
$$

Observe que aqui usamos a condição $p>2$.

Consideraremos, agora, o outro caso, ou seja, $0<r<t-\rho$.

Considerando-se $Q=p(p-2)-1$, notamos que $Q>0$, pois tal hipótese impõe $p>1+\sqrt{2}$. Fazendo a mudança de variáveis $\alpha=s+\lambda, \beta=s-\lambda$, usando (1.26) e (1.27), temos

$$
\int_{0}^{t} d s \int_{|r-t+s|}^{r+t-s} \frac{\lambda}{2 r} \bar{u}^{p}(\lambda, s) d \lambda \leq \frac{c^{p} \rho^{p(p-1)}}{8 r} \int_{-\rho}^{t-r} d \beta \int_{t-r}^{t+r} \frac{\alpha-\beta}{(\alpha+2 \rho)^{p}(\beta+2 \rho)^{p(p-2)}} d \alpha
$$




$$
\leq \frac{c^{p} \rho^{p(p-1)}}{8 r} \int_{-\rho}^{\infty} d \beta \int_{t-r}^{t+r} \frac{d \alpha}{(\alpha+2 \rho)^{p-1}(\beta+2 \rho)^{Q+1}}=\frac{c^{p} \rho^{p+1}}{8 r Q} \int_{t-r}^{t+r}(\alpha+2 \rho)^{1-p} d \alpha .
$$

Trabalhando separadamente os casos $(t-r+2 \rho)>\frac{1}{2}(t+r+2 \rho)$ e $(t-r+2 \rho)<$ $\frac{1}{2}(t+r+2 \rho)$, sempre chegamos a

$$
\int_{0}^{t} d s \int_{|r-t+s|}^{r+t-s} \frac{\lambda}{2 r} \bar{u}^{p}(\lambda, s) d \lambda \leq \frac{2 c^{p} \rho^{p+1}}{Q(p-2)(t+r+2 \rho)(t-r+2 \rho)^{p-2}} .
$$

Desta forma, prova-se o lema.

Nossa definição de norma, aplicada a funções que satisfaçam (1.26), implica que

$$
\begin{gathered}
|u(t, x)| \leq\|u\| \\
\left\||u|^{\theta}|v|^{1-\theta}\right\| \leq\|u\|^{\theta}\|v\|^{1-\theta} \quad, \quad 0 \leq \theta \leq 1 .
\end{gathered}
$$

Vejamos que (1.29) com (1.25), implicam

$$
L\left(|u|^{\theta p}|v|^{(1-\theta) p}\right) \leq C \rho^{2}\|u\|^{\theta p}\|v\|^{(1-\theta) p} \quad, \quad 0 \leq \theta \leq 1
$$

Denotemos por $Z$ o espaço linear de funções $u(t, x)$, tais que $D^{\alpha} u(t, x)$ para $|\alpha| \leq 2$ estão definidas e são contínuas para todo $(t, x) \in \mathbb{R}_{+}^{1+3}$ e satisfaçam

$$
u(t, x)=0, \quad\left|x-x^{0}\right|>t+\rho
$$

e

$$
\left\|D^{\alpha} u\right\|<\infty \quad, \quad|\alpha| \leq 2 .
$$

Em particular percebemos de imediato que $u^{0} \in Z$.

Por outro lado, se $u \in Z$, vemos que a partir de (1.23) e (1.30) (usando $\theta=(p-1) / p$ )

$$
\left\|D_{k} L|u|^{p}\right\| \leq p\left\|L\left(|u|^{p-1}\left|D_{k} u\right|\right)\right\|=p\left\|L\left(|u|^{\theta p} D_{k}|u|^{(1-\theta) p}\right)\right\| \leq C p \rho^{2}\|u\|^{p-1}\left\|D_{k} u\right\| .
$$

Similarmente, derivando espacialmente duas vezes e aplicando em um termo o que foi feito anteriormente, e no outro termo utilizando primeiro (1.30) $\operatorname{com} \theta=(p-2) / p$, seguido de (1.29) $\operatorname{com} \theta=1 / 2$, chegamos a

$$
\left\|D_{j} D_{k} L|u|^{p}\right\| \leq C p \rho^{2}\left\{(p-1)\|u\|^{p-2}\left\|D_{j} u\right\|\left\|D_{k} u\right\|+\|u\|^{p-1}\left\|D_{j} D_{k} u\right\|\right\} .
$$


Agora, definindo $w(t, x)=\max (|u(t, x)|,|v(t, x)|)$ e $\|w\|=\max (\|u\|,\|v\|)$, para $u, v \in$ $Z$, podemos demonstrar que

$$
\begin{gathered}
\left\|L\left\{|u|^{p}-|v|^{p}\right\}\right\| \leq C p \rho^{2}\|w\|^{p-1}\|u-v\| \\
\left\|D_{j} L\left\{|u|^{p}-|v|^{p}\right\}\right\| \leq C p(p-1) \rho^{2}\|w\|^{p-2}\|u-v\|\left\|D_{j} u\right\| \\
+C p \rho^{2}\|w\|^{p-2}\left\|D_{j}(u-v)\right\| \\
\left\|D_{j} D_{k} L\left\{|u|^{p}-|v|^{p}\right\}\right\| \leq \\
\leq C p(p-1) \rho^{2}\|w\|^{p-2}\left[\left\|D_{j} D_{k} u\right\|\|u-v\|\right. \\
\left.+\left\|D_{k} u\right\|\left\|D_{j}(u-v)\right\|+\left\|D_{j} v\right\|\left\|D_{k}(u-v)\right\|\right] \\
+C p \rho^{2}\|w\|^{p-1}\left\|D_{j} D_{k}(u-v)\right\| \\
+C p(p-1) \rho^{2}\|u-v\|^{p-2}\left\|D_{j} u\right\|\left\|D_{k} u\right\| .
\end{gathered}
$$

Através de (1.23), (1.31) e (1.32) deduzimos diretamente que $L|u|^{p} \in Z$ sempre que $u \in Z$.

Chegamos à parte final da prova, pois o nosso processo iterativo mencionado será feito ao se definir a seqüência de funções $\left\{u_{n}\right\}$ da seguinte forma:

$$
u_{0}=u^{0} \quad, \quad u_{n+1}=u_{0}+L\left|u_{n}\right|^{p} \quad, \quad n \geq 0 .
$$

A partir dessa definição com (1.23), segue que

$$
\left\|u_{n+1}\right\| \leq\left\|u^{0}\right\|+C \rho^{2}\left\|u^{n}\right\|^{p}
$$

Assumamos agora que $\left\|u^{0}\right\|$ é tão pequena que ${ }^{3}$

$$
C \rho^{2}\left\|u^{0}\right\|^{p-1} \leq \frac{1}{p 2^{p}}, \quad\left\|u^{0}\right\|<\frac{1}{2} .
$$

Com esta hipótese podemos aplicar indução sobre (1.37), obtendo

$$
\left\|u^{n}\right\| \leq 2\left\|u^{0}\right\|<1 \quad, \quad n \geq 0
$$

Daqui em diante $C_{i}, i=1,2, \ldots$ denotarão constantes que dependem de $p, \rho$ e $\left\|D^{\alpha} u^{0}\right\|$, para $|\alpha| \leq 2$, mas não de $n$.

A partir de (1.38), (1.31) e (1.39) deduzimos diretamente

$$
\left\|D_{k} u_{n+1}\right\| \leq\left\|D_{k} u^{0}\right\|+\frac{1}{2}\left\|D_{k} u_{n}\right\| .
$$

\footnotetext{
${ }^{3}$ Através de (1.22) essa condição é equivalente a que $N_{0}$ seja suficientemente pequeno.
} 
Logo,

$$
\left\|D_{k} u_{n}\right\| \leq C_{1} \quad, \quad n \geq 0
$$

De (1.32) constatamos que

$$
\begin{aligned}
\left\|D_{j} D_{k} u_{n+1}\right\| & \leq\left\|D_{j} D_{k} u_{0}\right\|+C p(p-1) \rho^{2} 2^{p-2}\left\|u^{0}\right\|^{p-2} C_{1}^{2}+C p \rho^{2} 2^{p-1}\left\|u^{0}\right\|^{p-1}\left\|D_{j} D_{k} u_{n}\right\| \\
& \leq C_{2}+\frac{1}{2}\left\|D_{j} D_{k} u_{n}\right\| .
\end{aligned}
$$

Logo,

$$
\left\|D_{j} D_{k} u_{n}\right\| \leq C_{3}
$$

Por último usamos (1.33), (1.34) e (1.35) com $u=u_{n}, v=u_{n-1}, w=\max \left(\left|u_{n}\right|,\left|u_{n-1}\right|\right)$, $\|w\| \leq 2\left\|u_{0}\right\|$. Feito isso, para $n \geq 1$ obtemos

$$
\left\|u_{n+1}-u_{n}\right\| \leq C p \rho^{2} 2^{p-1}\left\|u^{0}\right\|^{p-1}\left\|u_{n}-u_{n-1}\right\| \leq \frac{1}{2}\left\|u_{n}-u_{n-1}\right\|,
$$

e, assim,

$$
\left\|u_{n+1}-u_{n}\right\| \leq 2^{-n} C_{4}, \quad n \geq 0 .
$$

Da mesma forma, para $n \geq 1$ vale

$$
\begin{aligned}
&\left\|D_{j}\left(u_{n+1}-u_{n}\right)\right\| \leq C p(p-1) \rho^{2} 2^{p-2}\left\|u^{0}\right\|^{p-2}\left\|u_{n}-u_{n-1}\right\|\left\|D_{j} u_{0}\right\| \\
&+C p \rho^{2} 2^{p-1}\left\|u^{0}\right\|^{p-1}\left\|D_{j}\left(u_{n}-u_{n-1}\right)\right\| \\
& \leq 2^{-n} C_{5}+\frac{1}{2}\left\|u_{n}-u_{n-1}\right\|, \\
&\left\|D_{j}\left(u_{n+1}-u_{n}\right)\right\| \leq n 2^{-n} C_{6} \quad, \quad n \geq 0
\end{aligned}
$$

Finalmente fazendo contas similares, chegamos a

$$
\left\|D_{j} D_{k}\left(u_{n+1}-u_{n}\right)\right\| \leq\left(n 2^{-n}+2^{-(p-2) n}\right) C_{7}+\frac{1}{2}\left\|D_{j} D_{k}\left(u_{n}-u_{n-1}\right)\right\| .
$$

Assim,

$$
\left\|D_{j} D_{k}\left(u_{n+1}-u_{n}\right)\right\| \leq\left(n^{2} 2^{-n}+n 2^{-(p-2) n}\right) C_{8} .
$$

Dos resultados (1.42), (1.43) e (1.44) podemos concluir que $D^{\alpha} u_{n}$, para $|\alpha| \leq 2$ converge uniformemente para $D^{\alpha} u$, quando $n \rightarrow \infty$, sendo essas funções contínuas em $t$ e $x$. Portanto, $u$ é a solução do problema. Então, desta forma, consideramos demonstrado o teorema. 


\section{Estimativas de Strichartz}

Neste capítulo discutiremos as estimativas integrais $L^{p}-L^{q}$ obtidas por Robert S. Strichartz relacionadas com a equação da onda (ver [21], [22]).

\subsection{Convoluções com Núcleos que possuem suas singularidades sobre a esfera}

Os operadores de convolução da forma $T f(x)=\int_{\mathbb{R}^{n}} f(x-y) K(y) d y$, onde $K$ tem suas singularidades sobre a esfera unitária, aparecem naturalmente no estudo da equação da onda. Veremos agora que é possivel obter estimativas desses operadores com relação à sua limitação em várias normas $L^{p}$, as quais nos proporcionarão novas informações sobre a solução dessa equação.

Especificamente, denotemos por $\Sigma$ a esfera unitária em $\mathbb{R}^{n}, x, y$ denotarão pontos de $\mathbb{R}^{n}$ e $x^{\prime}, y^{\prime}$ denotarão pontos de $\Sigma$. Seja também $d x^{\prime}$ a medida de Lebesgue sobre $\Sigma$, de tal forma que $\int_{\mathbb{R}^{n}} f(x) d x=\int_{0}^{\infty} \int_{\Sigma} f\left(r x^{\prime}\right) d x^{\prime} r^{n-1} d r$.

Se $f \in C_{c}^{\infty}\left(\mathbb{R}^{n}\right)$ e $k$ é um inteiro não negativo, definimos

$$
T_{k+1} f(x)=\left.\int_{\Sigma}\left(\frac{\partial}{\partial r}\right)^{k} f\left(x-r y^{\prime}\right)\right|_{r=1} d y^{\prime} .
$$

Para $0<\alpha<1$ definimos

$$
T_{\alpha} f(x)=\int_{|y| \leq 1} f(x-y)\left(1-|y|^{2}\right)^{-\alpha} d y .
$$




\subsection{Convoluções com Núcleos que possuem suas singularidades sobre a esfera19}

Veja que a integral em (2.2) carece de sentido para $\alpha \geq 1$, pois o termo $\left(1-|y|^{2}\right)^{-\alpha}$ possui uma singularidade não integrável sobre $\Sigma$. Mas, interpretando (2.2) no sentido fraco, podemos definir $T_{\alpha}$ para valores não inteiros de $\alpha$ em $0<\alpha \leq(n+1) / 2$. Para tanto, integramos formalmente por partes em (2.2), omitindo os termos de fronteira. Isto é, se $k<\alpha<k+1$ definimos

$$
T_{\alpha} f=\left(-\frac{1}{2}\right)^{k} \frac{\Gamma(\alpha-k)}{\Gamma(\alpha)} \int_{\Sigma} \int_{0}^{1}\left(1-r^{2}\right)^{k-\alpha}\left(\frac{\partial}{\partial r} \frac{1}{r}\right)^{k}\left(r^{n-1} f\left(x-r y^{\prime}\right)\right) d r d y^{\prime} .
$$

Para esses operadores de convolução cujos núcleos possuem singularidades sobre a esfera, podemos demonstrar o seguinte:

Teorema 2.1 São válidas as seguintes estimativas:

(a)

$$
\left\|T_{\alpha} f\right\|_{q} \leq A_{\alpha}\|f\|_{p} \quad \text { para } \quad 0<\alpha \leq \frac{n+1}{2}
$$

sempre que

$$
1<p \leq 2 \leq q<\infty \quad \text { e } \quad \frac{1}{p}-\frac{1}{q} \leq \frac{n+1-2 \alpha}{2 n}
$$

(b)

$$
\left\|T_{\alpha} f\right\|_{p^{\prime}} \leq A_{\alpha}\|f\|_{p} \quad \text { para } \quad 0<\alpha \leq \frac{n+1}{2}
$$

sempre que

$$
p=\frac{n+1}{n+1-\alpha} \quad \text { e } \quad p^{\prime}=\frac{n+1}{\alpha} .
$$

(c)

$$
\left\|T_{\alpha} f\right\|_{q} \leq A_{\alpha}\|f\|_{p} \quad \text { para } \quad \frac{1}{2} \leq \alpha \leq \frac{n+1}{2}
$$

sempre que

$$
\frac{n+1}{n+1-\alpha} \leq p \leq 2 \quad \text { e } \frac{n}{q}=\alpha-\frac{1}{p}
$$

ou

$$
\frac{n}{n+\frac{1}{2}-\alpha} \leq p \leq \frac{n+1}{n+1-\alpha} \quad \text { e } \quad \frac{1}{q}=\alpha-\frac{n}{p^{\prime}},
$$

onde $p^{\prime}$ é o exponente conjugado de $p$, ou seja, $1 / p+1 / p^{\prime}=1$. 


\subsection{Convoluções com Núcleos que possuem suas singularidades sobre a esfera20}

Prova. Definindo a função

$$
\phi_{\alpha}(y)= \begin{cases}\left(1-|y|^{2}\right)^{-\alpha} & \text { se }|y|<1 \\ 0 & \text { se }|y| \geq 1\end{cases}
$$

notamos que para $\alpha<1, \phi_{\alpha} \in L^{1}$ e assim, podemos calcular sua Transformada de Fourier, isto é (ver [2])

$$
\widehat{\phi_{\alpha}}(\xi)=(2 \pi)^{-n / 2} 2^{-\alpha} \Gamma(1-\alpha)|\xi|^{\alpha-n / 2} J_{n / 2-\alpha}(|\xi|) .
$$

A prova desse teorema baseia-se no fato de que, a partir de (2.6), a família $T_{\alpha}$ de operadores pode ser caracterizada pelas seguintes expressões:

$$
\begin{gathered}
\widehat{T_{\alpha} f}(\xi)=(2 \pi)^{-n / 2} 2^{-\alpha} \Gamma(1-\alpha)|\xi|^{\alpha-n / 2} J_{n / 2-\alpha}(|\xi|) \widehat{f}(\xi) \quad \text { para } \alpha \neq 1,2, \ldots \\
\widehat{T_{k} f}(\xi)=\sum_{i=1}^{k} c_{i}|\xi|^{i-n / 2} J_{n / 2-i}(|\xi|) \widehat{f}(\xi) \quad \text { para } k=1,2,3, \ldots
\end{gathered}
$$

Assim, bastará demonstrar o teorema para a família de operadores $S_{\alpha}$, analítica na faixa $0 \leq \operatorname{Re}(\alpha) \leq \frac{n+1}{2}$

$$
\widehat{S_{\alpha} f}(\xi)=|\xi|^{\alpha-n / 2} J_{n / 2-\alpha}(|\xi|) \widehat{f}(\xi)
$$

$\operatorname{com} f \in C_{c}^{\infty}\left(\mathbb{R}^{n}\right)$.

Notemos que é suficiente a demonstração do teorema para $S_{\alpha}$ no lugar de $T_{\alpha}$.

Com esse objetivo utilizaremos a estimativa clássica para funções de Bessel complexas

$$
\left|t^{-(a+i b)} J_{a+i b}(t)\right| \leq C_{a} e^{c|b|}(1+t)^{-a-1 / 2},
$$

válida para todo real $0<t<\infty$.

Essa estimativa é suficiente para a demonstração da parte (a) do teorema como conseqüência da aplicação do teorema de Hardy e Littlewood, o qual garante que o operador $\mathscr{F}^{-1}(m(\xi) \widehat{f}(\xi))$ é limitado como operador de $L^{p}$ em $L^{q}$ sempre que $1<p \leq$ $2 \leq q<\infty$ e

$$
|m(\xi)| \leq c|\xi|^{-t} \quad \text { para } \frac{1}{p}-\frac{1}{q}=\frac{t}{n} .
$$

É evidente que $S_{\alpha}$ possui essa forma sendo $m(\xi)=|\xi|^{\alpha-n / 2} J_{n / 2-\alpha}(|\xi|)$; logo, considerando $\alpha$ real e aplicando $(2.9)$ nesse $m(\xi)$, obtemos

$$
|m(\xi)| \leq c|\xi|^{-\left(\frac{n+1}{2}-\alpha\right)} .
$$




\subsection{Convoluções com Núcleos que possuem suas singularidades sobre a esfera21}

Portanto, (2.10) é válida para todo $t \leq \frac{n+1}{2}-\alpha$, e, assim, (a) fica satisfatoriamente demonstrado.

$\mathrm{Na}$ prova do item (b) usaremos o teorema de interpolação complexa de Stein (ver apêndice A) aplicado a $S_{\alpha}$ Note-se que $S_{\alpha}$ é uma família analítica de operadores na faixa $0<\operatorname{Re}(\alpha)<(n+1) / 2$ e que também, devido à condição (2.9), tem crescimento admissível. Respeitando a notação utilizada no apêndice citado, consideremos $p_{1}=$ $q_{1}=2, p_{2}=1$ e $q_{2}=\infty$. Dessa forma os equivalentes das hipóteses (A.3) e (A.4) para esta família são

$$
\left\|S_{\alpha} f\right\|_{2} \leq c e^{c I m(\alpha)}\|f\|_{2}, \quad \text { para } \operatorname{Re}(\alpha)=\frac{n+1}{2}
$$

e

$$
\left\|S_{\alpha} f\right\|_{\infty} \leq c e^{c \operatorname{Im}(\alpha)}\|f\|_{1}, \quad \text { para } \operatorname{Re}(\alpha)=0,
$$

sendo claro que $c e^{c \operatorname{Im}(\alpha)}$ satisfaz (A.5).

A estimativa (2.11) segue diretamente de (2.9) e da aplicação do Teorema de Plancherel (ver apêndice C).

Para provar a validade da estimativa (2.12) usamos (2.7), o fato de que $\left\|T_{\alpha} f\right\|_{\infty} \leq$ $\|f\|_{1}$, pois $\left|\phi_{\alpha}(x)\right| \leq 1$ e a identidade $|\Gamma(1+i b)|=(\pi b / \sinh b)^{1 / 2}$.

Logo, todas as hipóteses do teorema de interpolação de Stein são satisfeitas. Na linguagem do apêndice referido temos $q_{1}=\infty, p_{1}=1, q_{2}=p_{2}=2$ e $t=2 \operatorname{Re}(\alpha) /(n+1)$. Assim, (A.2) se transforma em

$$
\frac{1}{p}=1-\frac{\operatorname{Re}(\alpha)}{2} \quad \text { e } \frac{1}{q}=\frac{\operatorname{Re}(\alpha)}{2} .
$$

Daí nota-se diretamente que $q=p^{\prime}$ e que substituindo o valor de $t$, obtemos o resultado procurado.

O item (c) é obtido como uma aplicação direta do Teorema de Interpolação real de M. Riesz (ver apêndice A), tendo como hipótese os items (a) e (b), e considerando $\alpha>1 / 2$.

No caso $\alpha=1 / 2$ verifica-se que $T_{1 / 2}$ opera de $L^{1}$ em $L_{2}^{*}$, pois $\phi_{1 / 2} \in L_{2}^{*}$. Portanto, nesse caso particular, o resultado segue da aplicação do Teorema de Interpolação real de Marcinkiewicz (ver apêndice A).

Dessa forma, concluimos a demonstração do teorema. 


\subsection{Convoluções com Núcleos que possuem suas singularidades sobre a esfera22}

O passo seguinte consiste em aplicar esse resultado à equação da onda em $n$ dimensões espaciais.

Sabemos que o problema de Cauchy

$$
\begin{gathered}
\square u(t, x)=\partial^{2} u(t, x) / \partial t^{2}-\Delta_{x} u(t, x)=0, \\
u(0, x)=f(x), \quad \partial u(0, x) / \partial t=g(x)
\end{gathered}
$$

para $f, g \in C_{c}^{\infty}\left(\mathbb{R}^{n}\right)$, tem solução única dada por

$$
u(t, x)=\mathscr{F}^{-1}\left(\widehat{f}(\xi) \cos t|\xi|+\widehat{g}(\xi) \frac{\sin t|\xi|}{|\xi|}\right),
$$

onde $\mathscr{F}^{-1}$ denota a Transformada Inversa de Fourier.

Observando as identidades para a função de Bessel

$$
J_{1 / 2}(t)=\left(\frac{2}{\pi}\right)^{1 / 2} \frac{\sin t}{t^{1 / 2}} \quad \text { e } J_{-1 / 2}(t)=\left(\frac{2}{\pi}\right)^{1 / 2} \frac{\cos t}{t^{1 / 2}},
$$

não é difícil ver que (2.15) pode ser escrita como

$$
u(t, x)=\left(\frac{\pi}{2}\right)^{1 / 2} \delta(t) S_{(n+1) / 2} \delta\left(t^{-1}\right) f+\left(\frac{\pi}{2}\right)^{1 / 2} t \delta(t) S_{(n-1) / 2} \delta\left(t^{-1}\right) g,
$$

onde $\delta(t)$ é o operador de dilatação

$$
[\delta(t) f](x)=f(t x)
$$

e $S_{(n+1) / 2}$ e $S_{(n-1) / 2}$ são os valores em $\alpha=(n+1) / 2$ e $\alpha=(n-1) / 2$ respectivamente da família (2.8).

Definimos a p-energia de $u$ por

$$
E_{p}(t)=\int_{\mathbb{R}^{n}}\left\{\left|\frac{\partial u}{\partial t}(t, x)\right|^{p}+\left|\nabla_{x} u(t, x)\right|^{p}\right\} d x .
$$

Veja que $E_{p}(0)=\|g\|_{p}^{p}+\|\nabla f\|_{p}^{p}$.

Observemos que o princípio clássico de conservação de energia diz que $E_{2}(t)$ é constante. Littman demonstrou que este resultado não tem seu análogo para $p \neq 2$ (ver [13]). De fato, nesse caso existem soluções fracas com energia finita para $t=0 \mathrm{e}$ energia infinita para todo $t \neq 0$.

O resultado seguinte nos fornece uma estimativa para a norma $L^{q}$ de $u(t, x)$, em termos da $p$-energia no tempo $t=0$ para certos valores de $p$ e $q$. 


\subsection{Convoluções com Núcleos que possuem suas singularidades sobre a esfera23}

Teorema 2.2 Seja $u(t, x)$ como em (2.15), $n \geq 2$; então, para qualquer $t \neq 0$

$$
\left(\int_{\mathbb{R}^{n}}|u(t, x)|^{q} d x\right)^{1 / q} \leq c(t) E_{p}(0)^{1 / p},
$$

onde

$$
\frac{n}{q}=\frac{n-1}{2}-\frac{1}{p^{\prime}} \quad \text { para } \quad 2 \frac{n+1}{n+3} \leq p \leq 2
$$

$e$

$$
\frac{1}{q}=\frac{n}{p}-\frac{n+1}{2} \quad \text { para } \quad 2 \frac{n}{n+2} \leq p \leq 2 \frac{n+1}{n+3}
$$

no caso $n=2$ considerar $p>1$.

Prova. Notemos que devido a (2.16), é suficiente provar que

$$
\left\|S_{(n-1) / 2} g\right\|_{q} \leq c\|g\|_{p}
$$

e

$$
\left\|S_{(n+1) / 2} f\right\|_{q} \leq c\|\nabla f\|_{p} .
$$

Agora, (2.22) é consequência do teorema (2.1), pois o valor $\alpha=(n-1) / 2$, substituído em (2.4) e (2.5), reproduz as hipóteses (2.20) e (2.21), respectivamente; e como já mencionamos, as mesmas estimativas são válidas tanto para $T_{\alpha}$ como para $S_{\alpha}$.

Veja que demonstrar (2.23) equivale a

$$
\left\|\mathscr{F}^{-1}\left(\frac{\cos |\xi|}{|\xi|} \widehat{f}(\xi)\right)\right\|_{q} \leq c\|f\|_{p} .
$$

Nesse sentido consideramos a função de corte $\psi \in C^{\infty}\left(\mathbb{R}^{n}\right)$, a qual se anula em uma vizinhança da origem e é igual a um para valores grandes de $\xi$.

Então, novamente o Teorema de Hardy-Littlewood implica.

$$
\left\|\mathscr{F}^{-1}\left((1-\psi(\xi)) \frac{\cos |\xi|}{|\xi|} \widehat{f}(\xi)\right)\right\|_{q} \leq c\|f\|_{p}
$$

sempre que $p$ e $q$ satisfaçam $1<p \leq 2 \leq q<\infty$ e $1 / p-1 / q \geq 1 / n$.

Somente resta estudar o seguinte operador:

$$
\left\|\mathscr{F}^{-1}\left(\psi(\xi) \frac{\cos |\xi|}{|\xi|} \widehat{f}(\xi)\right)\right\|_{q} \leq c\|f\|_{p} .
$$

Nesse ponto somente diremos que a prova dessa estimativa é muito similar à prova do teorema (2.1), ao considerar a família analítica de operadores

$$
U_{\alpha}^{(j)}(f)=\mathscr{F}^{-1}\left(\psi(\xi) \xi_{j}|\xi|^{\alpha-n / 2-1} J_{n / 2-\alpha-1}(|\xi|) \widehat{f}(\xi)\right) .
$$




\subsection{Estimativa a priori para a equação da onda}

Seja $\Omega=\left\{x \in \mathbb{R}^{n}:\left|x-x_{0}\right|<T\right\}$ uma bola no espaço $n$ dimensional e seja $\Gamma=$ $\left\{(t, x):\left|x-x_{0}\right|<T-|t|,|t|<T\right\}$ a união dos cones do passado e do futuro com base em $\Omega$. Consideraremos para $1<p<\infty$ os Espaços de Sobolev de ordem inteira $W^{k, p}(\Gamma)$ definidos no apêndice B. Como $\Gamma$ satisfaz as hipóteses do Teorema de Extensão de Calderón, (ver apêndice B), então, qualquer função em $W^{k, p}(\Gamma)$ pode ser definida fora de $\Gamma$, de tal forma que esteja em $W^{k, p}\left(\mathbb{R}^{1+n}\right)$.

Nesta seção estaremos interessados na solução do seguinte tipo de problema:

$$
\begin{gathered}
\square u=w \quad \text { em } \Gamma \\
u(0, \cdot)=u_{t}(0, \cdot)=0 \quad \text { em } \Omega,
\end{gathered}
$$

no sentido fraco, para $w \in W^{k, p}(\Gamma)$.

Teorema 2.3 Dado $w \in W^{k, p}(\Gamma)$, existe uma única solução $u \in W^{k, q}(\Gamma)$ do problema (2.24) e (2.25), onde $p=2(n+1) /(n+3), q=2(n+1) /(n-1)$, e

$$
\|u\|_{k, q, \Gamma} \leq M\|w\|_{k, p, \Gamma}
$$

para alguma constante $M$.

Prova. Comecemos considerando $w \in C_{c}^{\infty}\left(\mathbb{R}^{1+n}\right)$. Sabe-se que a solução do problema, nesse caso, pode ser expressada explicitamente através da transformada de Fourier. Assim, denotando por $\widehat{w}(t, \xi)$ e $\widehat{u}(t, \xi)$ as tranformadas de Fourier de $w$ e $u$ respectivamente, nas variáveis espaciais para um tempo fixo $t$, temos que

$$
\widehat{u}(t, \xi)=\int_{0}^{t} \frac{\sin (t-s)|\xi|}{|\xi|} \widehat{w}(s, \xi) d s
$$

é uma solução de (2.24) e (2.25), no sentido clássico, em todo $\mathbb{R}^{n}$.

$\mathrm{Na}$ seção anterior provamos a estimativa seguinte:

$$
\left\|\mathscr{F}^{-1}\left[\frac{\sin |\xi|}{|\xi|} \widehat{f}(\xi)\right]\right\|_{q} \leq M_{1}\|f\|_{p}
$$


Segue então, que

$$
\left\|\mathscr{F}^{-1}\left[\frac{\sin t|\xi|}{|\xi|} \widehat{f}(\xi)\right]\right\|_{q} \leq M_{1}|t|^{1+n((1 / q)-(1 / p))}\|f\|_{p}
$$

pois

$$
\mathscr{F}^{-1}\left[\frac{\sin t|\xi|}{|\xi|} \widehat{f}(\xi)\right]=t \delta\left(t^{-1}\right)\left(\mathscr{F}^{-1}\left\{\frac{\sin |\xi|}{|\xi|} \widehat{\delta(t) f}(\xi)\right\}\right)
$$

onde $\delta$ é o operador de dilatação definido em (2.17), e devido a que $\|\delta(t) f\|_{p}=$ $|t|^{-n / p}\|f\|_{p}$.

Aplicando (2.29) em (2.27) obtemos

$$
\|u(t, \cdot)\|_{q} \leq M_{1} \int_{0}^{t}|t-s|^{1+n((1 / q)-(1 / p))}\|w(s, \cdot)\|_{p} d s .
$$

Por outro lado observemos que os valores de $p$ e $q$ satisfazem

$$
1+n\left(\frac{1}{q}-\frac{1}{p}\right)=-\frac{n-1}{n+1}=-1+\frac{1}{p}-\frac{1}{q},
$$

isso nos permite aplicar o Teorema de Integração Fracionária devido a Hörmander (ver [7]). Logo, a convolução com $s^{1+n[(1 / q)-(1 / p)]}$ é um operador limitado de $L^{p}$ em $L^{q}$. Assim, obtemos a seguinte estimativa global nas variáveis temporal e espacial:

$$
\|u\|_{q} \leq M\|w\|_{p}
$$

Veja que por densidade a fórmula (2.27) também faz sentido para $w \in L^{p}\left(\mathbb{R}^{1+n}\right)$ e desta maneira nos fornece uma solução fraca de (2.24) e (2.25), a qual, pelo mesmo argumento, satisfaz (2.31). A estimativa local (2.26), para $k=0$, é obtida extendendo $w$ nulamente fora de $\Gamma$ e aplicando a estimativa global (2.31).

Nosso objetivo então, é demonstrar (2.26) para $k>0$. Perceba que para tanto é suficiente dominar

$$
\left\|\partial^{k} u / \partial x_{j}^{k}\right\|_{L^{q}(\Gamma)} \text { e }\left\|\partial^{k} u / \partial t^{k}\right\|_{L^{q}(\Gamma)} \text { por }\|w\|_{k, p, \Gamma}
$$

Como $w \in W^{k, p}(\Gamma)$ vemos que trabalhar com as derivadas espaciais é fácil, pois

$$
\square \partial^{k} u / \partial x_{j}^{k}=\partial^{k} w / \partial x_{j}^{k} \in L^{p}(\Gamma) \text { e } \quad \partial^{k} u / \partial x_{j}^{k}
$$

tem dados de Cauchy nulos. Assim, temos todas as hipóteses para aplicar (2.26) com $k=0$. 
O trabalho com as derivadas temporais não pode ser feito de modo análogo, pois essas derivadas não satisfazem mais os dados de Cauchy do problema. No entanto, a expressão do operador de D'Alambert nos proporciona uma pista de como devemos agir neste caso. Assim, já que $\partial^{2} u / \partial t^{2}=\Delta_{x} u+w$, podemos expressar qualquer derivada de ordem par, $\partial^{k} u / \partial t^{k}$, como combinação linear de derivadas espaciais de $u$, de ordem $k$, e derivadas de $w$ de ordem $\leq k-2$. Pela Desigualdade de Sobolev essas derivadas de $w$ estão em $L^{q}(\Gamma)$, pois $(1 / p)-(1 / q)=2 /(n+1)$. Logo, a estimativa desejada para $\partial^{k} u / \partial t^{k}$ é valida se $k$ for par. Se $k$ for ímpar, o argumento anterior mostra que $\partial^{k-1} u / \partial t^{k-1}=D_{x} u+v$ para $v \in W^{1, q}(\Gamma)$ e onde $D_{x}$ representa um operador diferencial de ordem $k-1$ nas variáveis espaciais. Como $\partial v / \partial t \in$ $L^{q}(\Gamma)$ somente resta mostrar que $(\partial / \partial t) D_{x} u \in L^{q}(\Gamma)$. Pois bem, veja que $\square D_{x} u=$ $D_{x} w \in W^{1, q}(\Gamma)$, logo, podemos estender $D_{x} w$ fora de $\Gamma$ de tal forma que esteja em $W^{1, q}\left(\mathbb{R}^{1+n}\right)$. Agora, como $D_{x} u$ tem dados de Cauchy nulos, podemos aplicar (2.26), assim, sendo $u_{0}$ alguma extensão de $u$ para $\mathbb{R}^{1+n}$ obtemos

$$
\widehat{D_{x} u_{0}}(t, \xi)=\int_{0}^{t} \frac{\sin (t-s)|\xi|}{|\xi|} \widehat{D_{x} w}(s, \xi) d s .
$$

Derivando com relação a $t$ e chamando

$$
\widehat{w_{0}}(t, \xi)=|\xi| \widehat{D_{x} w}(t, \xi)
$$

obtemos

$$
\frac{\partial}{\partial t} \widehat{D_{x} u_{0}}(t, \xi)=\int_{0}^{t} \frac{\cos (t-s)|\xi|}{|\xi|} \widehat{w_{0}}(s, \xi) d s .
$$

De (2.32) segue que $w_{0} \in L^{q}$, daí que podemos repetir o feito desde (2.26) até (2.31) para (2.33), levando em consideração que na seção anterior também foi provada uma estimativa similar à (2.28) substituindo sin por cos. Portanto obtemos

$$
\left\|(\partial / \partial t) D_{x} u\right\|_{L^{q}(\Gamma)} \leq M\left\|w_{0}\right\|_{L^{q}(\Gamma)}
$$

com o qual completamos a prova do teorema.

Faremos um pequeno comentário sobre a escolha dos valores particulares de $p$ e $q$ no último teorema. Na seção anterior provamos (2.28) para diversos valores de $p$ e $q$, aqui somente usamos aqueles valores que maximizam a diferença $(1 / p)-(1 / q)$, sendo esse máximo $2 /(n+1)$. Realmente é uma coincidência que com esses valores específicos seja possivel aplicar o Teorema de Integração Fracionária à expressão (2.30). 


\section{Existência Global em $n$ Dimensões}

\section{Espaciais}

\subsection{Formulação do Problema e o Teorema Central}

Consideraremos para uma dada potência $p>1$ termos não lineares $F_{p}$ que satisfaçam

$$
\left|\left(\frac{\partial}{\partial u}\right)^{j} F_{p}(u)\right| \leq C_{j}|u|^{p-j}, \quad j=0,1 .
$$

De fato, o caso modelo é $F_{p}(u)=|u|^{p}$. Se $\mathbb{R}_{+}^{1+n}=\mathbb{R}_{+} \times \mathbb{R}^{n}$, e se $f, g \in C_{c}^{\infty}\left(\mathbb{R}^{n}\right)$ são fixos, estudaremos o seguinte tipo de problema de Cauchy:

$$
\left\{\begin{array}{l}
\square u=F_{p}(u), \quad(t, x) \in \mathbb{R}_{+}^{1+n} \\
u(0, x)=\varepsilon f(x), \quad \partial_{t} u(0, x)=\varepsilon g(x),
\end{array}\right.
$$

onde $\square=\frac{\partial^{2}}{\partial t^{2}}-\Delta_{x}$ representa o operador de D'Alambert em $n$ dimensões espaciais. Estamos interessados em achar, para $n$ dado, o domínio preciso de potências para as quais possamos sempre obter uma solução fraca global de (3.2), se $\varepsilon>0$ for suficientemente pequeno. A resposta está intimamente relacionada com a potência crítica $p_{c}$, a qual é solução da seguinte equação quadrática:

$$
(n-1) p_{c}^{2}-(n+1) p_{c}-2=0 \quad, \quad p_{c}>1 .
$$

Estudaremos especificamente a resposta dada por Georgiev, Lindblad e Sogge (ver [4]) que se resume no seguinte teorema, o qual constitui o centro desta dissertação: 
Teorema 3.1 Seja $n \geq 3$ e assuma que $F_{p}$, satisfazendo (3.1), é fixo com $p_{c}<p \leq$ $\frac{n+3}{n-1}$. Então, se $\varepsilon>0$ for suficientemente pequeno, (3.2) tem uma única solução (fraca) global u, tal que

$$
\left(1+\left.\left|t^{2}-\right| x\right|^{2} \mid\right)^{\gamma} u \in L^{p+1}\left(\mathbb{R}_{+}^{1+n}\right)
$$

para algum $\gamma$ que satisfaça

$$
\frac{1}{p(p+1)}<\gamma<\frac{(n-1) p-(n+1)}{2(p+1)} .
$$

Note-se que como $n \geq 3$ a parábola $y=(n-1) x^{2}-(n+1) x-2$ sempre possui duas raízes reais e distintas (uma negativa e outra estritamente maior do que um), então, a potência crítica pedida em (3.3) existe. Portanto, a condição (3.5) imposta sobre $\gamma$ somente faz sentido se $p>p_{c}$, pois $p>1$ e

$$
\frac{1}{p(p+1)}<\frac{(n-1) p-(n+1)}{2(p+1)} \Longleftrightarrow 0<(n-1) p^{2}-(n+1) p-2
$$

Por outro lado, veja que no teorema são consideradas potências menores que a potência conformemente invariante $p_{\text {conf }}=\frac{n+3}{n-1}$, pois sabe-se que para potências maiores que $p_{\text {conf }}$, a existência global está garantida (ver [11]).

Provaremos o teorema usando certas estimativas de Strichartz com pesos para a solução da equação da onda não homogênea

$$
\left\{\begin{array}{l}
\square w(t, x)=F(t, x), \quad(t, x) \in \mathbb{R}_{+}^{1+n} \\
0=w(0, \cdot)=\partial_{t}(0, \cdot),
\end{array}\right.
$$

as quais estão estabelecidas no seguinte teorema:

Teorema 3.2 Suponha que $n \geq 2$ e que w resolve a equação da onda linear não homogênea (3.6), onde o termo forçante satisfaça $F(t, x)=0$ se $t-|x| \leq 1$. Então,

$$
\left\|\left(t^{2}-|x|^{2}\right)^{\gamma_{1}} w\right\|_{L^{q}\left(\mathbb{R}_{+}^{1+n}\right)} \leq C_{q, \gamma}\left\|\left(t^{2}-|x|^{2}\right)^{\gamma_{2}} F\right\|_{L^{q /(q-1)}\left(\mathbb{R}_{+}^{1+n}\right)},
$$

sempre que $2 \leq q \leq \frac{2(n+1)}{n-1}$, e

$$
\gamma_{1}<n\left(\frac{1}{2}-\frac{1}{q}\right)-\frac{1}{2} \quad \text { e } \gamma_{2}>\frac{1}{q}
$$


A estimativa (3.7) pode ser pensada como uma versão com pesos das estimativas de Strichartz (ver [22]) para (3.6), isto é, uma versão com pesos de

$$
\|w\|_{L^{2(n+1) /(n-1)}\left(\mathbb{R}_{+}^{1+n}\right)} \leq C\|F\|_{L^{2(n+1) /(n+3)}\left(\mathbb{R}_{+}^{1+n}\right)} .
$$

Por ser a estimativa (3.7) justamente a chave na demonstração do teorema principal (3.1) da nossa dissertação, deixaremos sua demonstração para o capítulo seguinte.

\subsection{Processo Iterativo}

Reescalonando a variável temporal por $R>0$, obtemos a seguinte versão equivalente à estimativa (3.7):

$$
\begin{aligned}
\left\|\left((t+R)^{2}-|x|^{2}\right)^{\gamma_{1}} w\right\|_{L^{q}\left(\mathbb{R}_{+}^{1+n}\right)} & \leq C_{q, \gamma}\left\|\left((t+R)^{2}-|x|^{2}\right)^{\gamma_{2}} F\right\|_{L^{q /(q-1)}\left(\mathbb{R}_{+}^{1+n}\right)} \\
\text { se } F(t, x) & =0,|x| \geq t+R-1 ;
\end{aligned}
$$

aqui, $q$ e $\gamma_{j}(j=1,2)$ se apresentam como no teorema (3.2).

Utilizaremos esta versão na demonstração do teorema (3.1) mediante a prova de um processo iterativo, assim resumido:

Lema 3.3 Seja $u_{-1} \equiv 0$, e para $m=0,1,2,3, \ldots$, definamos $u_{m}$ recursivamente por

$$
\left\{\begin{array}{l}
\square u_{m}=F_{p}\left(u_{m-1}\right) \\
u_{m}(0, x)=\varepsilon f(x) \quad, \quad \partial_{t} u_{m}(0, x)=\varepsilon g(x),
\end{array}\right.
$$

onde $f, g \in C_{c}^{\infty}\left(\mathbb{R}^{n}\right)$ são fixos e se anulam fora da bola de raio $R-1(R>1)$, com centro na origem. Ademais, considerando $p_{c}<p \leq \frac{n+3}{n-1}$, $\gamma$ tal que $\frac{1}{p(p+1)}<\gamma<$ $\frac{(n-1) p-(n+1)}{2(p+1)}$ e definindo

$$
\begin{aligned}
& A_{m}=\left\|\left((t+R)^{2}-|x|^{2}\right)^{\gamma} u_{m}\right\|_{L^{p+1}\left(\mathbb{R}_{+}^{1+n}\right)} \\
& B_{m}=\left\|\left((t+R)^{2}-|x|^{2}\right)^{\gamma}\left(u_{m}-u_{m-1}\right)\right\|_{L^{p+1}\left(\mathbb{R}_{+}^{1+n}\right)}
\end{aligned}
$$

então, existe $\varepsilon_{0}>0$ que depende de $p, F_{p}, \gamma$ e dos dados $f, g$, tal que para $m=$ $0,1,2, \ldots$

$$
A_{m} \leq 2 A_{0} \text { e } 2 B_{m+1} \leq B_{m}, \text { se } \varepsilon<\varepsilon_{0}
$$


Prova. Devido às condições impostas sobre o suporte dos dados, considerações sobre o domínio de dependência implicam que $u_{m}$ e também $F_{p}\left(u_{m}\right)$ devem se anular se $|x|>t+R-1$. Sabe-se também que a solução $u_{0}$ da equação da onda $\square u_{0}=0 \mathrm{com}$ os dados anteriores (ver [8]), satisfaz

$$
u_{0}=O\left(\varepsilon(1+t)^{-(n-1) / 2}(1+|t-| x||)^{-(n-1) / 2}\right)
$$

designando por $\left.\Gamma=\left\{(t, x) \in \mathbb{R}_{+}^{1+n}\right) /|x| \leq t+R-1\right\}$, temos

$$
\begin{aligned}
A_{0} & =\left\|\left((t+R)^{2}-|x|^{2}\right)^{\gamma} u_{0}\right\|_{L^{p+1}\left(\mathbb{R}_{+}^{1+n}\right)} \\
& =\left[\int_{0}^{\infty} \int_{\Gamma}\left\{\left.\left.\left|(t+R)^{2}-\right| x\right|^{2}\right|^{\gamma}\left|u_{0}(t, x)\right|\right\}^{p+1} d x d t\right]^{1 /(p+1)} \\
& \leq M \varepsilon\left[\int_{0}^{\infty} \int_{\Gamma}\left\{\left.\left.\left|(t+R)^{2}-\right| x\right|^{2}\right|^{\gamma}(1+t)^{-(n-1) / 2}(1+|t-| x||)^{-(n-1) / 2}\right\}^{p+1} d x d t\right]^{1 /(p+1)} .
\end{aligned}
$$

Por outro lado, é fácil verificar que para $(t, x) \in \Gamma$ são válidas

$$
\begin{aligned}
& (t+R)^{2}-|x|^{2} \geq 1, \\
& (1+t)^{-(n-1) / 2}<C_{1}(t+R+|x|)^{-(n-1) / 2}, \\
& (1+|t-| x||)^{-(n-1) / 2}<C_{2}(t+R-|x|)^{-(n-1) / 2},
\end{aligned}
$$

onde $C_{1}$ e $C_{2}$ são constantes que dependem de $R$ e $n$.

Também sabemos que $\gamma<\frac{(n-1) p-(n+1)}{2(p+1)}$. Logo, existe um $\delta>0$, tal que $\gamma=$ $\frac{(n-1) p-(n+1)}{2(p+1)}-\delta$. Isso somado ao que foi exposto anteriormente nos leva à

$$
A_{0} \leq M C_{1} C_{2} \varepsilon[\underbrace{\int_{0}^{\infty} \int_{\Gamma}(t+R-|x|)^{-n-\delta(p+1)}(t+R+|x|)^{-n-\delta(p+1)} d x d t}_{\mathbf{I}}]^{1 /(p+1)} .
$$

Fazendo uma mudança de variável para coordenadas polares $x=r \omega, \omega \in S^{n-1} \mathrm{e}$ $d x=r^{n-1} d r d \omega$ e estimando superiormente obtemos

$$
\begin{aligned}
I & =\int_{0}^{\infty} \int_{|\omega|=1} \int_{0}^{t+R-1}(t+R-r)^{-n-\delta(p+1)}(t+R+r)^{-n-\delta(p+1)} r^{n-1} d r d \omega d t \\
& =\int_{|\omega|=1} \int_{0}^{\infty} \int_{0}^{t+R-1} \frac{r^{n-1}}{(t+R+r)^{n-1}} \cdot \frac{1}{(t+R+r)^{1+\delta(p+1)}(t+R-r)^{n+\delta(p+1)}} d r d t d \omega \\
& \leq \int_{|\omega|=1}^{\infty} \int_{0}^{\infty} \frac{1}{(t+R)^{1+\delta(p+1)}} \int_{0}^{t+R-1} \frac{d r}{(t+R-r)^{n+\delta(p+1)}} d t d \omega .
\end{aligned}
$$


Na última integral em $r$ fazemos $\xi=t+R-r-1$, resultando

$$
\int_{0}^{t+R-1} \frac{d r}{(t+R-r)^{n+\delta(p+1)}}=\int_{0}^{t+R-1} \frac{d \xi}{(1+\xi)^{n+\delta(p+1)}} \leq \int_{0}^{\infty} \frac{d \xi}{(1+\xi)^{n+\delta(p+1)}}=K .
$$

Assim, a integral $I$ é convergente, pois a esfera $S^{n-1}$ é compacta, e a integral $\int_{0}^{\infty} \frac{d t}{(t+R)^{1+\delta(p+1)}}$ é, de fato, convergente.

Concluindo, chegamos à importante estimativa

$$
A_{0} \leq C_{0} \varepsilon
$$

Para $j, m>0$ vale

$$
\begin{aligned}
\square\left(u_{m+1}-u_{j+1}\right) & =\square u_{m+1}-\square u_{j+1}=F_{p}\left(u_{m}\right)-F_{p}\left(u_{j}\right) \\
& =\int_{0}^{1} \frac{d}{d \tau} F_{p}\left(\tau u_{m}+(1-\tau) u_{j}\right) d \tau \\
& =\left(u_{m}-u_{j}\right) \int_{0}^{1} \frac{\partial}{\partial u} F_{p}\left(\tau u_{m}+(1-\tau) u_{j}\right) d \tau \\
& =\left(u_{m}-u_{j}\right) V_{p}\left(u_{m}, u_{j}\right) .
\end{aligned}
$$

Pois bem, lembrando a condição (3.1) imposta sobre $F_{p}$, concluimos que

$$
\square\left(u_{m+1}-u_{j+1}\right)=V_{p}\left(u_{m}, u_{j}\right)\left(u_{m}-u_{j}\right), \quad \text { onde } \quad V_{p}\left(u_{m}, u_{j}\right)=O\left(\left(\left|u_{m}\right|+\left|u_{j}\right|\right)^{p-1}\right) .
$$

Este problema tem dados de Cauchy iguais a zero devido aos dados de Cauchy do problema (3.10). Além disso, fazendo $q=p+1$, nossas hipóteses sobre $\gamma$ e $p$, resultam em

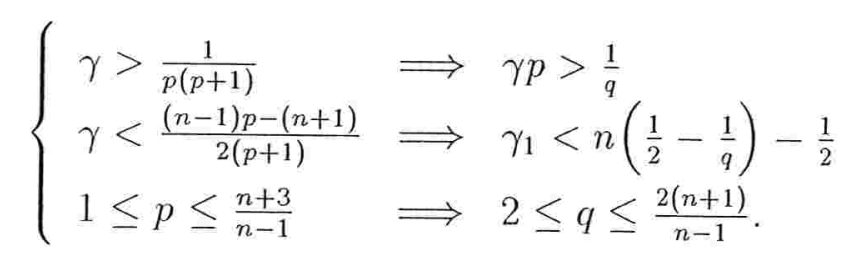

É evidente que podemos aplicar a este problema o teorema (3.2) na versão (3.9) com $\gamma_{1}=\gamma, \gamma_{2}=\gamma p$ e $q=p+1$, já que para todo $m$ e $j, u_{m}-u_{j}$ anula-se quando $|x|>t+R-1$. Assim, obtemos

$$
\begin{aligned}
& \left\|\left((t+R)^{2}-|x|^{2}\right)^{\gamma}\left(u_{m+1}-u_{j+1}\right)\right\|_{L^{p+1}} \\
& \quad \leq C_{1}\left\|\left((t+R)^{2}-|x|^{2}\right)^{p \gamma} V_{p}\left(u_{m}, u_{j}\right)\left(u_{m}-u_{j}\right)\right\|_{L^{(p+1) / p}} \\
& \quad=C_{1}\left\|\left((t+R)^{2}-|x|^{2}\right)^{(p-1) \gamma} V_{p}\left(u_{m}, u_{j}\right)\left((t+R)^{2}-|x|^{2}\right)^{\gamma}\left(u_{m}-u_{j}\right)\right\|_{L^{(p+1) / p}} \\
& \quad \leq C_{1}\left\|\left((t+R)^{2}-|x|^{2}\right)^{(p-1) \gamma} V_{p}\left(u_{m}, u_{j}\right)\right\|_{L^{(p+1) /(p-1)}}\left\|\left((t+R)^{2}-|x|^{2}\right)^{\gamma}\left(u_{m}-u_{j}\right)\right\|_{L^{p+1}} .
\end{aligned}
$$


A passagem anterior resultou de uma aplicação direta da Desigualdade de Hölder (ver Apêndice C), pois $\frac{p}{p+1}=\frac{1}{r}+\frac{1}{p+1}$ implica $r=\frac{p+1}{p-1}$. Agora, estimando a primeira norma e lembrando que $V_{p}\left(u_{m}, u_{j}\right)=O\left(\left(\left|u_{m}\right|+\left|u_{j}\right|\right)^{p-1}\right)$, chegamos a

$$
\begin{aligned}
\|\left((t+R)^{2}\right. & \left.-|x|^{2}\right)^{(p-1) \gamma} V_{p}\left(u_{m}, u_{j}\right) \|_{L^{(p+1) /(p-1)}} \\
& \leq C_{2}\left\|\left((t+R)^{2}-|x|^{2}\right)^{\gamma}\left|u_{m}\right|+\left((t+R)^{2}-|x|^{2}\right)^{\gamma}\left|u_{j}\right|\right\|_{L^{(p+1) /(p-1)}}^{p-1} \\
& \leq C_{2}\left(\left\|\left((t+R)^{2}-|x|^{2}\right)^{\gamma} u_{m}\right\|_{L^{p+1}}+\left\|\left((t+R)^{2}-|x|^{2}\right)^{\gamma} u_{j}\right\|_{L^{p+1}}\right)^{p-1} .
\end{aligned}
$$

Substituindo essa estimativa pela principal resulta

$$
\begin{aligned}
\left\|\left((t+R)^{2}-|x|^{2}\right)^{\gamma}\left(u_{m+1}-u_{j+1}\right)\right\|_{L^{p+1}} & \\
\leq & C\left(\left\|\left((t+R)^{2}-|x|^{2}\right)^{\gamma} u_{m}\right\|_{L^{p+1}}+\left\|\left((t+R)^{2}-|x|^{2}\right)^{\gamma} u_{j}\right\|_{L^{p+1}}\right)^{p-1} \\
& \quad \times\left\|\left((t+R)^{2}-|x|^{2}\right)^{\gamma}\left(u_{m}-u_{j}\right)\right\|_{L^{p+1}} .
\end{aligned}
$$

Portanto, pela definição de $A_{m}$, essa estimativa se resume em

$$
\left\|\left((t+R)^{2}-|x|^{2}\right)^{\gamma}\left(u_{m+1}-u_{j+1}\right)\right\|_{L^{p+1}} \leq C\left(A_{m}+A_{j}\right)^{p-1}\left\|\left((t+R)^{2}-|x|^{2}\right)^{\gamma}\left(u_{m}-u_{j}\right)\right\|_{L^{p+1}} .
$$

A partir desse resultado, demonstraremos a primeira parte do lema por indução. Para. $m=0$ é óbvio que $A_{0} \leq 2 A_{0}$; só resta provar que se $A_{k} \leq 2 A_{0}$, então $A_{k+1} \leq 2 A_{0}$, para $\varepsilon \leq \varepsilon_{0}$.

Como $u_{-1} \equiv 0$, temos que $A_{-1}=0$; assim, fazendo $j=-1$ em (3.13) é valida

$$
\left\|\left((t+R)^{2}-|x|^{2}\right)^{\gamma}\left(u_{k+1}-u_{0}\right)\right\|_{L^{p+1}} \leq C A_{k}^{p-1}\left\|\left((t+R)^{2}-|x|^{2}\right)^{\gamma} u_{k}\right\|_{L^{p+1}} .
$$

Daí, $A_{k+1} \leq A_{0}+C A_{k}^{p-1} A_{k}$; mas pela nossa hipótese indutiva $A_{k} \leq 2 A_{0}$. Além disso, como (3.12), afirma que $A_{0} \leq C_{0} \varepsilon$, podemos tomar um $\varepsilon_{0}$, tal que para $\varepsilon<\varepsilon_{0}$ : $A_{k}^{p-1} \leq\left(2 C_{0} \varepsilon_{0}\right)^{p-1}$. Porém, esse $\varepsilon_{0}$ pode ser tão pequeno que $C\left(2 C_{0} \varepsilon_{0}\right)^{p-1} \leq 1 / 2$; assim, $A_{k+1} \leq A_{0}+\frac{1}{2} 2 A_{0}=2 A_{0}$. Demonstramos, dessa forma, a primeira parte do lema.

Provemos a segunda parte. Considerando em (3.13), $j=k-1$ e $m=k$ temos $\left\|\left((t+R)^{2}-|x|^{2}\right)^{\gamma}\left(u_{k+1}-u_{k}\right)\right\|_{L^{p+1}} \leq C\left(A_{k}+A_{k-1}\right)^{p-1}\left\|\left((t+R)^{2}-|x|^{2}\right)^{\gamma}\left(u_{k}-u_{k-1}\right)\right\|_{L^{p+1}}$, ou seja, $B_{k+1} \leq C\left(A_{k}+A_{k-1}\right)^{p-1} B_{k}$. Pela primeira parte do lema, já provada, tem-se que existe $\varepsilon_{0}$, tal que para $\varepsilon<\varepsilon_{0}, A_{k} \leq 2 A_{0} \leq 2 C_{0} \varepsilon_{0}$, logo $B_{k+1} \leq C\left(4 C_{0} \varepsilon_{0}\right)^{p-1} B_{k}$. 
Por último, podemos tomar $\varepsilon_{0}$ suficientemente pequeno, tal que $C\left(4 C_{0} \varepsilon_{0}\right)^{p-1} \leq \frac{1}{2}$, para assim concluir, como queríamos demonstrar, que $2 B_{k+1} \leq B_{k}$.

Assim, o lema fica integralmente demonstrado.

\subsection{Demonstração do Teorema Central}

Veremos nesta seção como é possível a demonstração do teorema (3.1) a partir do lema (3.3).

Prova. Comecemos provando a parte de existência.

Se $\varepsilon>0$ em (3.2) for pequeno, os $u_{m}$ forem como em (3.10) e considerando $m>n$, $m=n+k$, temos

$$
\begin{aligned}
\left\|u_{m}-u_{n}\right\|_{L^{p+1}} & \leq\left\|u_{n+k}-u_{n+k-1}\right\|_{L^{p+1}}+\ldots+\left\|u_{n+1}-u_{n}\right\| \\
& \leq B_{n+k}+\ldots+B_{n+1} \text { pois }\left((t+R)^{2}-|x|^{2}\right)^{\gamma} \geq 1 .
\end{aligned}
$$

Conforme provamos antes $2 B_{m+1} \leq B_{m}$ e $B_{0}=A_{0} \leq C_{0} \varepsilon_{0}$; logo, para $\varepsilon<\varepsilon_{0}$ é fácil deduzir que $B_{m} \leq \frac{1}{2^{m}} A_{0}$. Tal dedução, ligada à estimativa anterior, nos fornece

$$
\left\|u_{m}-u_{n}\right\|_{L^{p+1}} \leq\left(\frac{1}{2^{n}}-\frac{1}{2^{m}}\right) C_{0} \varepsilon_{0} .
$$

Isto é, $\left\{u_{m}\right\}$ é uma sequência de Cauchy em $L^{p+1}\left(\mathbb{R}_{+}^{1+n}\right)$, o qual é um espaço completo, daí existir um $u \in L^{p+1}\left(\mathbb{R}_{+}^{1+n}\right)$, tal que $u_{m} \rightarrow u$ em $L^{p+1}\left(\mathbb{R}_{+}^{1+n}\right)$; e é claro que essa convergência também se dá no sentido fraco.

Vimos que $F_{p}\left(u_{m+1}\right)-F_{p}\left(u_{m}\right)=V_{p}\left(u_{m+1}, u_{m}\right)\left(u_{m+1}-u_{m}\right)$, onde $V_{p}\left(u_{m+1}, u_{m}\right)=$ $O\left(\left(\left|u_{m+1}\right|+\left|u_{m}\right|\right)^{p-1}\right)$, então

$$
\left|F_{p}\left(u_{m+1}\right)-F_{p}\left(u_{m}\right)\right| \leq C_{1}\left(\left(\left|u_{m+1}\right|+\left|u_{m}\right|\right)^{p-1}\right)\left|u_{m+1}-u_{m}\right|
$$


Com isso podemos estimar a seguinte expressão:

$$
\begin{aligned}
&\left\|F_{p}\left(u_{m+1}\right)-F_{p}\left(u_{m}\right)\right\|_{L^{(p+1) / p}} \\
& \leq C_{1}\left\|\left(\left|u_{m+1}\right|+\left|u_{m}\right|\right)^{p-1}\left(u_{m+1}-u_{m}\right)\right\|_{L^{(p+1) / p}} \\
& \leq C_{1}\left\|\left(\left|u_{m+1}\right|+\left|u_{m}\right|\right)^{p-1}\right\|_{L^{(p+1) / p}}\left\|\left(u_{m+1}-u_{m}\right)\right\|_{L^{p+1}} \\
& \leq C_{1}\left\|\left|u_{m+1}\right|+\left|u_{m}\right|\right\|_{L^{p+1}}^{p-1}\left\|\left(u_{m+1}-u_{m}\right)\right\|_{L^{p+1}} \\
& \leq C_{1}\left(\left\|\left((t+R)^{2}-|x|^{2}\right)^{\gamma} u_{m+1}\right\|_{L^{p+1}}+\left\|\left((t+R)^{2}-|x|^{2}\right)^{\gamma} u_{m}\right\|_{L^{p+1}}\right)^{p-1} \\
& \times\left\|\left((t+R)^{2}-|x|^{2}\right)^{\gamma}\left(u_{m+1}-u_{m}\right)\right\|_{L^{p+1}} \\
& \leq C_{1}\left(A_{m+1}+A_{m}\right)^{p-1} B_{m+1} .
\end{aligned}
$$

Nesse cálculo usamos tanto a desigualdade de Hölder como o fato $\left((t+R)^{2}-|x|^{2}\right)^{\gamma} \geq 1$. Logo, utilizando as já conhecidas estimativas para $A_{m}$ e $B_{m}$, obtemos

$$
\left\|F_{p}\left(u_{m+1}\right)-F_{p}\left(u_{m}\right)\right\|_{L^{(p+1) / p}}=O\left(\frac{1}{2^{m}}\right) .
$$

Essa última, por sua vez, nos leva a concluir que

$$
\left\|F_{p}\left(u_{m}\right)-F_{p}\left(u_{n}\right)\right\|_{L^{(p+1) / p}} \leq K\left(\frac{1}{2^{n}}-\frac{1}{2^{m}}\right) .
$$

Assim, provamos que a seqüência $\left\{F_{p}\left(u_{m}\right)\right\}$ é de Cauchy em $L^{(p+1) / p}\left(\mathbb{R}_{+}^{1+n}\right)$, o qual é um espaço completo; isto é, existe $V \in L^{(p+1) / p}$, tal que $F_{p}\left(u_{m}\right) \rightarrow V$. Portanto, passando ao limite em (3.10) e considerando $\varepsilon$ suficientemente pequeno, provamos a existência da solução fraca.

Provaremos a unicidade dessa solução e deixaremos para o final a demonstração de que essa, de fato, satisfaz (3.4).

Demonstraremos, então, que $V=F_{p}(u)$. Como vimos anteriormente, podemos chegar à expressão

$$
\left|F_{p}\left(u_{m}\right)-F_{p}(u)\right| \leq C\left(\left(\left|u_{m}\right|+|u|\right)^{p-1}\right)\left|u_{m}-u\right| .
$$

Logo,

$$
\begin{aligned}
\left\|F_{p}\left(u_{m}\right)-F_{p}(u)\right\|_{L^{(p+1) / p}} & \leq C\left\|\left(\left|u_{m}\right|+|u|\right)^{p-1}\left(u_{m}-u\right)\right\|_{L^{(p+1) / p}} \\
& \leq C\left(\left\|\left|u_{m}\right|\right\|_{L^{(p+1)}}\|+\||u| \|_{L^{(p+1)}}\right)^{p-1}\left\|\left(u_{m+1}-u\right)\right\|_{L^{p+1}} .
\end{aligned}
$$

Assim, como $u_{m} \rightarrow u$ em $L^{p+1}\left(\mathbb{R}_{+}^{1+n}\right)$, temos também que $F_{p}\left(u_{m}\right) \rightarrow F_{p}(u)$ em $L^{(p+1) / p}\left(\mathbb{R}_{+}^{1+n}\right)$ e, portanto, $V=F_{p}(u)$, como queríamos demonstrar. 
Finalmente como $A_{m} \leq 2 A_{0} \leq 2 C_{0} \varepsilon_{0}$, tomando limite na expressão do $A_{m}$, tem-se que

$$
\left\|\left((t+R)^{2}-|x|^{2}\right)^{\gamma} u\right\|_{L^{p+1}} \leq 2 C_{0} \varepsilon_{0} .
$$

Isso relacionado ao fato de que $1+\left.\left|t^{2}-\right| x\right|^{2}|\leq|(t+R)^{2}-|x|^{2} \mid$ para $R>1$, demonstra que, para $\varepsilon$ suficientemente pequeno, existe uma única solução fraca global do problema (3.2), tal que

$$
\left((t+R)^{2}-|x|^{2}\right)^{\gamma} u \in L^{p+1}\left(\mathbb{R}_{+}^{1+n}\right) .
$$

Eis, portanto, a nossa demonstração cabal do teorema principal. 


\section{Estimativas de Strichartz com}

\section{Pesos}

Neste capítulo daremos as idéias principais para a demonstração do Teorema (3.2). Para isso devemos centrar nosso trabalho na prova da estimativa (3.4).

\subsection{Roteiro da Demonstração}

Notemos que devido ao Teorema de Interpolação complexa de Stein (ver apêndice A), basta provar tal estimativa para os casos $q=2(n+1) /(n-1)$ e $q=2$. Observemos que para esses valores $q /(q-1)$ vale $2(n+1) /(n+3)$ e 2 respectivamente. Especificando, devemos provar que para $n \geq 2$

$$
\begin{aligned}
\left\|\left(t^{2}-|x|^{2}\right)^{\gamma_{1}} w\right\|_{L^{2(n+1) /(n-1)\left(\mathbb{R}_{+}^{1+n}\right)}} \leq & C_{\gamma}\left\|\left(t^{2}-|x|^{2}\right)^{\gamma_{2}} F\right\|_{L^{2(n+1) /(n+3)}\left(\mathbb{R}_{+}^{1+n}\right)}, \\
& \text { se } \gamma_{1}<(n-1) / 2(n+1)<\gamma_{2},
\end{aligned}
$$

e que

$$
\left\|\left(t^{2}-|x|^{2}\right)^{-\gamma} w\right\|_{L^{2}\left(\mathbb{R}_{+}^{1+n}\right)} \leq C_{\gamma}\left\|\left(t^{2}-|x|^{2}\right)^{\gamma} F\right\|_{L^{2}\left(\mathbb{R}_{+}^{1+n}\right)}, \quad \text { se } \gamma>1 / 2,
$$

sempre que $F(t, x)=0$ quando $t-|x|<1$.

Observemos que (4.1) é uma versão com pesos da Desigualdade de Strichartz (2.26). A estimativa $L^{2}$ seguirá essencialmente de uma dupla aplicação do Teorema do Traço 
de Sobolev.

Para a prova de (4.1) aproveitaremos as hipóteses feitas sobre os suportes de $w$ e $F$ e o fato de que os pesos são bem comportados. Desta maneira, temos que sobre as hipóteses anteriores $t^{2}-|x|^{2} \geq t$. Com esse fato, concluiremos que basta demonstrar uma variante de (4.1) na qual a norma da esquerda é tomada sobre faixas diádicas, onde $T / 2 \leq t \leq T$ para $T$ grande. Fazendo isso, temos que nossa estimativa se divide em duas partes. A parte mais simples está relacionada com a contribuição da $F$ para $w$ em, digamos, $t \geq T / 10$. Neste caso, usando geometria elementar e o fato de que os pesos são invariantes por Transformações de Lorentz, é possivel reduzir esse problema à demonstração de uma estimativa que segue diretamente do cálculo em $L^{2}$ de Operadores Integrais de Fourier (ver [6]). A análise sobre as contribuições da $F$ para tempos relativamente pequenos é muito mais complexo, pois os operadores integrais de Fourier que nela aparecem, se degeneram progressivamente a medida que $T \rightarrow \infty$, assim, nesse caso, não é possivel aplicar o teorema de Hörmander. Afortunadamente, esses operadores têm sido estudados por Sogge e Stein em [16], trabalho no qual os pesos são usados para compensar a degeneração desses operadores. Estes resultados somente podem ser usados no trabalho com a alta freqüência desses operadores, e é por isso que a demonstração da segunda parte é mais complexa do que a primeira. Para trabalhar com a baixa freqüência são usados algumas propriedaades geométricas e estimativas de fase estacionária.

A continuação, somente estudaremos a parte mais simples, antes mencionada, da demonstração de (4.1), sugerindo ao leitor interessado a revisão de [4] para os outros casos.

\subsection{Contribuição de $F$ para tempos relativamente grandes}

Como mencionamos na seção anterior, devido a que os pesos que aparecem na parte esquerda de (4.1) são menores que os pesos da direita, podemos reduzir nosso problema a demonstrar estimativas onde as normas da esquerda sejam tomadas sobre 
conjuntos, para os quais $t$ e $t-|x|$ estejam em intervalos diádicos. Assim, consideremos o caso para o qual $T / 2 \leq t \leq T$, para algum $T \geq 2$, e tal que $(t, x)$ satisfaça $|x| \leq t / 2$. Nosso primeiro passo será estabelecer a validez de uma variante da estimativa (4.1).

Proposição 4.1 Sejam $n \geq 2$ e $q=2(n+1) /(n-1)$, e assuma que $F(t, x)=0$ quando $t^{2}-|x|^{2} \leq 1$. Então se w é a solução da equação da onda não homogênea $\square w=F$ em $\mathbb{R}_{+}^{1+n}$ com dados de Cauchy nulos em $t=0$,

$$
\left\|\left(t^{2}-|x|^{2}\right)^{1 / q} w\right\|_{L^{q}(\{(t, x):|x| \leq t / 2, T / 2 \leq t \leq T\})} \leq C(\log T)^{1 / q}\left\|\left(t^{2}-|x|^{2}\right)^{1 / q} F\right\|_{L^{q /(q-1)}},
$$

onde $C$ depende somente da dimensão e $T \geq 2$.

Prova. Nesta demonstração apenas levaremos em conta a dimensão espacial ímpar. A demonstração para dimensões pares requer algumas formalidades adicionais, mas os resultados também são válidos.

Sejam $w_{T}(t, x)=w(T t, T x)$ e $F_{T}(t, x)=T^{2} F(T t, T x)$, dessa forma obtemos $\square w_{T}=$ $F_{T}$. De fato, provar (4.3) é equivalente a provar

$$
\left\|w_{T}\right\|_{L^{q}(\{(t, x):|x| \leq t / 2,1 / 2 \leq t \leq 1\})} \leq C(\log T)^{1 / q}\left\|\left(t^{2}-|x|^{2}\right)^{1 / q} F_{T}\right\|_{L^{q /(q-1)}} .
$$

Notemos que $F_{T}=0$ se $t^{2}-|x|^{2} \leq 1 / T^{2}$, também levando em consideração o domínio de dependência, podemos assumir que $F_{T}(t, x)=0$ se $t<1 / 8$. Devido às considerações realizadas, basta mostrar (4.4) assumindo que $F_{T}$ se anula para $t^{2}-|x|^{2} \leq 1 / T^{2}$ ou $t \leq 1 / 8$. As dificuldades na demonstração aparecem no momento em que consideramos $(t, x)$ perto do cone de luz, pois nesse caso os pesos na direita da estimativa são pequenos. Ademais, ao considerar $t-|x| \geq 1 / 8$ no suporte de $F_{T}$, vemos que a estimativa segue diretamente da versão sem pesos das estimativas de Strichartz (2.26). Portanto, podemos assumir para provar (4.3) que

$$
F_{T}(t, x)=0 \text { se } t \leq 1 / 8, \text { ou } t^{2}-|x|^{2} \leq 1 / T^{2} \text {, ou } t-|x| \geq 1 / 8
$$

Estabelecemos a última hipótese com o intuito de garantir que a diferença $t-s$ seja limitada inferiormente quando $t \geq s,(s, y) \in \operatorname{supp}\left(F_{T}\right)$ e tomando $(t, x)$ como no 
lado esquerdo de (4.4).

Agora, demonstraremos a parte principal da estimativa. Lembrando que $\widehat{F}(s, \xi)$ representa a transformada de Fourier na variável espacial, temos

$$
w_{T}(t, x)=(2 \pi)^{-n} \int_{0}^{t} \int_{\mathbb{R}^{n}} e^{i x \cdot \xi}|\xi|^{-1} \sin ((t-s)|\xi|) \widehat{F}_{T}(s, \xi) d \xi d s .
$$

Logo, se considerarmos a família

$$
\left(W^{z} F_{T}\right)(t, x)=(z-(n+1) / 2) e^{z^{2}} \int_{0}^{t} \int_{\mathbb{R}^{n}} e^{i x \cdot \xi}|\xi|^{-z} \sin ((t-s)|\xi|) \widehat{F}_{T}(s, \xi) d \xi d s .
$$

por interpolação complexa, será suficiente demonstrar

$$
\left\|W^{z} F_{T}\right\|_{L^{\infty}(|x| \leq t / 2,1 / 2 \leq t \leq 1)} \leq C\left\|F_{T}\right\|_{L^{1}}, \operatorname{Re}(z)=(n+1) / 2
$$

e

$$
\left\|W^{z} F_{T}\right\|_{L^{2}(|x| \leq t / 2,1 / 2 \leq t \leq 1)} \leq C(\log (T))^{1 / 2}\left\|\left(t^{2}-|x|^{2}\right)^{1 / 2} F_{T}\right\|_{L^{2}}, \operatorname{Re}(z)=0 .
$$

Como $t-s$ é limitada inferiormente, a estimativa (4.6) segue diretamente da seguinte:

$$
\left.\left|y e^{-y^{2}} \int e^{i x \cdot \xi+i t|\xi|}\right| \xi\right|^{-(n+1) / 2+i y} d \xi \mid \leq C_{n} t^{-(n-1) / 2}
$$

Na prova da estimativa $L^{2}$ note-se que $W^{z}=\left(W_{+}^{z}-W_{-}^{z}\right) / 2 i$, onde

$$
\begin{aligned}
\left(W_{ \pm}^{z} F\right)(t, x)= & (z-(n+1) / 2) e^{z^{2}} \\
& \times \iiint e^{i(x-y) \cdot \xi \pm i(t-s)|\xi|} F_{T}(s, y)|\xi|^{-z} d \xi d y d s \\
= & (z-(n+1) / 2) e^{z^{2}} \\
& \times \int_{1 / T}^{1 / 8} \iint e^{i(x-y) \cdot \xi \pm i(t-\tau-|y|)|\xi|} F_{T}(|y|+\tau, y)|\xi|^{-z} d \xi d y d \tau
\end{aligned}
$$

Aqui, estamos assumindo que $(t, x)$ é tomado como no lado esquerdo de $(4.7)$, logo, $s$ é menor que $t$ no suporte do primeiro integrando. Além disso, pela desigualdade de Hölder (ver apêndice C), a última expressão é dominada por $(\log T)^{1 / 2}$ vezes

$$
\left(\left.\left.\int\left|\tau^{1 / 2}(z-(n+1) / 2) e^{z^{2}} \iint e^{i(x-y) \cdot \xi \pm i(t-\tau-|y|)|\xi|} F_{T}(|y|+\tau, y)\right| \xi\right|^{-z} d \xi d y\right|^{2} d \tau\right)^{1 / 2} \text {. }
$$

Já que $\tau=s-|y|$ concluimos que é suficiente provar que para $\tau \leq 1 / 8$ e $1 / 2 \leq t \leq 1$ são válidas as seguintes cotas uniformes:

$\left\|\widetilde{W_{ \pm}^{z}} f(t-\tau, \cdot)\right\|_{L^{2}(\{x:|x| \leq t / 2\})} \leq C\|f\|_{L^{2}}, \operatorname{Re}(z)=0, \operatorname{supp}(f) \subset\{y: 1 / 8 \leq|y|<t-\tau\}$, 
se

$$
\left(\widetilde{W_{ \pm}^{z}} f\right)(t-\tau, x)=(z-(n+1) / 2) e^{z^{2}} \iint e^{i(x-y) \cdot \xi \pm i(t-\tau-|y|)|\xi|} f(y)|\xi|^{-z} d \xi d y
$$

Devido a (4.5) assumimos que $f$ se anula para $|y|<1 / 8$.

Como a fase

$$
\varphi_{ \pm}=(x-y) \cdot \xi \pm(t-\tau-|y|)|\xi|
$$

satisfaz para $\xi \neq 0$

$$
\operatorname{det} \partial^{2} \varphi_{ \pm} / \partial y_{j} \partial \xi_{k} \neq 0 \text { e } \nabla_{y} \varphi_{ \pm} \neq 0, \text { se } \nabla_{\xi} \varphi_{ \pm}=0
$$

a estimativa (4.9) deriva de uma aplicação direta do teorema de Hörmander (ver [6]). Com isso demonstramos a proposição.

Vejamos como é possível usar (4.3) para estimar $w$ se a norma é tomada sobre o conjunto onde $T / 2 \leq t \leq T$ e $F(t, x)$ se anula quando $t$ for menor que algum múltiplo prefixado de $T$. Lembremos que na hipótese do teorema (3.2) vale a condição $|x|<$ $t-1$ no suporte da $F$.

Especificando, se $w=w^{1}+w^{0}$, onde $\square w^{1}=F^{1}$ com dados de Cauchy nulos, e se $F^{1}(t, x)=F(t, x)$ para $t \geq T / 10$ e zero em outro caso, afirmamos que, para $q=2(n+1) /(n-1)$,

$$
\begin{aligned}
& \left\|\left(t^{2}-|x|^{2}\right)^{1 / q-\varepsilon} w^{1}\right\|_{L^{q}(\{(t, x): T / 2 \leq t \leq T\})} \\
& \quad \leq C T^{-2 \varepsilon}(\log T)^{2 / q}\left\|\left(t^{2}-|x|^{2}\right)^{1 / q+\varepsilon} F^{1}\right\|_{L^{q /(q-1)}} .
\end{aligned}
$$

Notemos que tanto $w^{1}$ e $F^{1}$ como $w$ e $F$ se anulam quando $t-|x| \leq 1$.

Procedemos a refinar a estimativa na variável $t-|x|$. Nesse sentido percebamos que (4.10) é conseqüência da desigualdade:

$$
\left\|\left(t^{2}-|x|^{2}\right)^{1 / q} w^{1}\right\|_{L^{q}\left(T / 2 \leq t \leq T, 2^{k-1} \leq t-|x| \leq 2^{k}\right)} \leq C(\log T)^{1 / q}\left\|\left(t^{2}-|x|^{2}\right)^{1 / q} F^{1}\right\|_{L^{q /(q-1)}}
$$

aqui é necessário impor que $2^{k} \leq 4 T$.

Por sua vez mostrar (4.11) é equivalente a provar

$$
\left\|\left(t^{2}-|x|^{2}\right)^{1 / q} w_{k}^{1}\right\|_{L^{q}\left(T_{k} / 2 \leq t \leq T_{k}, 1 / 2 \leq t-|x| \leq 1\right)} \leq C(\log T)^{1 / q}\left\|\left(t^{2}-|x|^{2}\right)^{1 / q} F_{k}^{1}\right\|_{L^{q /(q-1)}} .
$$


onde $T_{k}=T / 2^{k}, w_{k}^{1}(t, x)=w^{1}\left(2^{k} t, 2^{k} x\right)$ e $F_{k}^{1}(t, x)=2^{2 k} F^{1}\left(2^{k} t, 2^{k} x\right)$.

Finalmente diremos que (4.12) é obtida como resultado da aplicação de certas estimativas, as quais provêm de propriedades da solução fundamental do Operador de D'Alembert e da métrica Lorentziana. 


\section{Teoremas de Interpolação}

\section{A.1 Teorema de Interpolação de M. Riesz}

Seja $(U, \mu)$ um espaço de medida, sendo $\mu$ sempre uma medida positiva. Denotamos por $L_{p}(U, d \mu)$ o espaço de Lebesgue de todas as classes de equivalência de funções $\mu$-mensuráveis de valores complexos sobre $U$, tais que

$$
\|f\|_{L_{P}}=\left(\int_{U}|f|^{p} d \mu\right)^{1 / p}
$$

é finita. Aqui $1 \leq p<\infty$. No caso limite, $p=\infty, L_{p}$ está formado por todas as funções $\mu$-mensuráveis e limitadas. Assim, escrevemos

$$
\|f\|_{L_{\infty}}=\sup _{U}|f(x)| .
$$

Seja $T$ um operador linear de $L_{p}=L_{p}(U, d \mu)$ em $L_{q}=L_{q}(V, d \nu)$. Isso significa que $T(\alpha f+\beta g)=\alpha T(f)+\beta T(g)$. Escreveremos

$$
T: L_{p} \rightarrow L_{q}
$$

se, em adição, $T$ é limitado, isto é, se

$$
M=\sup _{f \neq 0} \frac{\|T f\|_{L_{q}}}{\|f\|_{L_{p}}}
$$

é finita. Chama-se $M$ a norma do operador $T$. 
Teorema A.1 Assuma que $p_{0} \neq p_{1}, q_{0} \neq q_{1}$ e que

$$
T: L_{p_{0}}(U, d \mu) \rightarrow L_{q_{0}}(V, d \nu)
$$

com norma $M_{0}$, e que

$$
T: L_{p_{1}}(U, d \mu) \rightarrow L_{q_{1}}(V, d \nu)
$$

com norma $M_{1}$. Então,

$$
T: L_{p}(U, d \mu) \rightarrow L_{q}(V, d \nu)
$$

com norma

$$
M \leq M_{0}^{1-\theta} M_{1}^{\theta}
$$

sempre que $0<\theta<1$ e

$$
\frac{1}{p}=\frac{1-\theta}{p_{0}}+\frac{\theta}{p_{1}}, \quad \frac{1}{q}=\frac{1-\theta}{q_{0}}+\frac{\theta}{q_{1}} .
$$

\section{A.2 Teorema de Interpolação de Marcinkiewicz}

Considere novamente o espaço de medida $(U, d \mu)$. Se $f$ é uma função $\mu$-mensurável de valores complexos (ou reais) finita, exceto em um conjunto de medida nula, introduzimos a função de distribuição $m(\sigma, f)$ definida por

$$
m(\sigma, f)=\mu(\{x:|f(x)|>\sigma\}) .
$$

Desde que assumamos que $\mu$ é positiva, $m(\sigma, f)$ será uma função de valores reais (ou reais extendidos) de $\sigma$, definida sobre o eixo real positivo $\mathbb{R}^{+}=(0, \infty)$. Está claro que $m(\sigma, f)$ é não crescente. Além disso, temos

$$
\|f\|_{L_{p}}=\left(p \int_{0}^{\infty} m(\sigma, f) \frac{d \sigma}{\sigma}\right)^{1 / p} \text { se } 1 \leq p<\infty
$$

e

$$
\|f\|_{L_{\infty}}=\inf \{\sigma: m(\sigma, f)=0\} .
$$

Usando essa função de distribuição, introduzimos os espaços $L_{p}$ fracos denotados por $L_{p}^{*}$. O espaço $L_{p}^{*}, 1 \leq p<\infty$ está formado por todas as $f$, tais que

$$
\|f\|_{L_{p}^{*}}=\sup _{\sigma} \sigma m(\sigma, f)^{1 / p}<\infty
$$


No caso limite $p=\infty$, tomamos $L_{\infty}^{*}=L_{\infty}$. Note-se que $\|f\|_{L_{p}^{*}}$ não é uma norma se $1 \leq p<\infty$. No entanto, para $p>1$ é possível definir uma norma em $L_{p}^{*}$ que o torna um Espaço de Banach (ver [1]). A relação

$$
\|f\|_{L_{p}}=\sup _{\sigma}\left(\int_{|f(x)|>\sigma}|f|^{p} d \mu\right)^{1 / p} \geq \sup _{\sigma} \sigma m(\sigma, f)^{1 / p}=\|f\|_{L_{p}^{*}}
$$

implica diretamente que $L_{p} \subset L_{p}^{*}$.

Por um operador linear limitado de $L_{p}$ em $L_{q}^{*}$ entenderemos um operador linear entre esses espaços que satisfaz $\|T f\|_{L_{q}^{*}} \leq C\|f\|_{L_{p}}$ e chamaremos norma de $T$ ao ínfimo do conjunto de todas aquelas constantes $C$. Todo esse comentário resume-se em $T: L_{p} \rightarrow L_{q}^{*}$.

Teorema A.2 Consideremos $p_{0} \neq p_{1}$ e que

$$
T: L_{p_{0}}(U, d \mu) \rightarrow L_{q_{0}^{*}}(V, d \nu)
$$

com norma $M_{0}^{*}$, e que

$$
T: L_{p_{1}}(U, d \mu) \rightarrow L_{q_{1}^{*}}(V, d \nu)
$$

com norma $M_{1}^{*}$.

Para $0<\theta<1$ consideremos

$$
\frac{1}{p}=\frac{1-\theta}{p_{0}}+\frac{\theta}{p_{1}}, \quad \frac{1}{q}=\frac{1-\theta}{q_{0}}+\frac{\theta}{q_{1}}
$$

e assumamos que

$$
p \geq q
$$

Então,

$$
T: L_{p}(U, d \mu) \rightarrow L_{q}(V, d \nu)
$$

com norma $M$, tal que

$$
M \leq C_{\theta}\left(M_{0}^{*}\right)^{1-\theta}\left(M_{1}^{*}\right)^{\theta} .
$$

\section{A.3 Teorema de Interpolação de Stein}

Enunciaremos o célebre resultado obtido por Elias M. Stein (ver [18]).

Diremos que uma função $\Phi(z)$ analítica na faixa aberta $0<\operatorname{Re}(z)<1$ e contínua na 
faixa fechada tem crescimento admissível se

$$
\sup _{|y| \leq r} \sup _{0 \leq x \leq 1} \log |\Phi(x+i y)| \leq A e^{a r}, \quad \text { para } a<\pi .
$$

Dados dois espaços de medida $M$ e $N$ com medidas $d m$ e $d n$ respectivamente e uma família de operadores lineares $T_{z}$ ( $z$ é um parâmetro complexo), diremos tratar-se de uma familia analítica de operadores se ela tiver as seguintes propriedades:

- Para cada $z, T_{z}$ é um operador linear de funções simples sobre $M$ em funções mensuráveis sobre $N$. Entenda-se por função simples sobre $M$ aquelas funções que possuem no máximo um número finito de valores não nulos quando atuam sobre conjuntos de medida finita de $M$.

- Sendo $\psi$ uma função simples sobre $M$ e $\phi$ uma função simples sobre $N$, então, $\Phi(z)=\int T_{z}(\psi) \phi d n$ é analítica em $0<\operatorname{Re}(z)<1$ e contínua em $0 \leq \operatorname{Re}(z) \leq 1$.

Também diremos que a família $T_{z}$ tem crescimento admissível, se $\Phi(z)=\int T_{z}(\psi) \phi d n$ tem crescimento admissível no sentido de (A.1). Esclarecemos, no entanto, que as constantes $A$ e $a$ podem depender de $\psi$ e $\phi$.

Teorema A.3 Seja $T_{z}$ uma família analítica de operadores lineares com crescimento admissível definida na faixa $0 \leq R e(z) \leq 1$. Suponhamos que

$$
1 \leq p_{1}, p_{2}, q_{1}, q_{2} \leq \infty
$$

e que

$$
\frac{1}{p}=\frac{1-t}{p_{1}}+\frac{t}{p_{2}}, \quad \frac{1}{q}=\frac{1-t}{q_{1}}+\frac{t}{q_{2}} \quad \text { onde } 0 \leq t \leq 1 .
$$

Ademais, para qualquer função simples $f$ são válidas

$$
\left\|T_{i y}(f)\right\|_{q_{1}} \leq A_{0}(y)\|f\|_{p_{1}}
$$

$e$

$$
\left\|T_{1+i y}(f)\right\|_{q_{2}} \leq A_{1}(y)\|f\|_{p_{2}}
$$

Finalmente impomos que

$$
\log \left|A_{i}(y)\right| \leq A e^{a|y|}, \quad a<\pi \text { para } i=0,1 .
$$


Daí, podemos concluir que

$$
\left\|T_{t}(f)\right\|_{q} \leq A_{t}\|f\|_{p}
$$

onde

$$
\log A_{t}=\int_{-\infty}^{+\infty} w(1-t, y) \log A_{0}(y) d y+\int_{-\infty}^{+\infty} w(t, y) \log A_{1}(y) d y
$$

e w é definido por

$$
w(t, y)=\frac{1 / 2 \tan (\pi t / 2)}{\left[\tan ^{2}(\pi t / 2)+\tanh ^{2}(\pi y / 2)\right] \cosh ^{2}(\pi y / 2)} .
$$




\section{Teorema de Extensão de Calderón}

\section{B.1 Espaços de Sobolev de Ordem Inteira}

Definiremos estes espaços sobre um domínio arbitrário $\Omega \subset \mathbb{R}^{n}$ como subespaços de diversos espaços $L^{p}(\Omega)$.

Definição B.1 Dado um número inteiro não negativo $m e 1 \leq p \leq \infty$, definimos o funcional $\|\cdot\|_{m, p}$, como segue:

$$
\begin{aligned}
& \|u\|_{m, p}=\left\{\sum_{0 \leq|\alpha| \leq m}\left\|D^{\alpha} u\right\|_{p}^{p}\right\}^{1 / p} \text { se } 1 \leq p<\infty \\
& \|u\|_{m, \infty}=\max _{0 \leq|\alpha| \leq m}\left\|D^{\alpha} u\right\|_{\infty},
\end{aligned}
$$

onde u é qualquer função para a qual o lado direito da definição faz sentido e $\|\cdot\|_{p}$. representa a norma em $L^{p}(\Omega)$.

Notemos que em ambos os casos estamos definindo uma norma sobre qualquer espaço vetorial de funções, onde o lado direito é finito, lembrando que funções nesses espaços devem ser identificadas no sentido $\mu$-q.s..

Definição B.2 Definimos o espaço

$$
W^{m, p}(\Omega) \equiv\left\{u \in L^{p}(\Omega): D^{\alpha} u \in L^{p}(\Omega) \text { para } 0 \leq|\alpha| \leq m\right\}
$$

onde as derivadas são tomadas no sentido fraco. 
O espaço $W^{m, p}(\Omega)$ munido da sua respectiva norma, dada na definição (B.1), é chamado de Espaço de Sobolev.

Pode-se demonstrar que $W^{m, p}(\Omega)$ é um espaço de Banach e, caso for necessário, sua norma será denotada por $\|u\|_{m, p, \Omega}$ no lugar de $\|u\|_{m, p}$.

Definição B.3 Dados $m$ e $p$, diremos que um operador $E l$, agindo de $W^{m, p}(\Omega)$ em $W^{m, p}\left(\mathbb{R}^{n}\right)$, é um $(m, p)$-operador de extensão simples para $\Omega$, se existir uma constante $K=K(m, p)$, tal que para qualquer $u \in W^{m, p}(\Omega)$, as seguintes condições são satisfeitas:

1. $E u(x)=u(x) \quad \mu$-q.s. em $\Omega$,

2. $\|E u\|_{m, p, \mathbb{R}^{n}} \leq K\|u\|_{m, p, \Omega}$.

A existência de um $(m, p)$-operador de extensão simples para um domínio $\Omega$, garante que $W^{m, p}(\Omega)$ herde várias das propriedades que $W^{m, p}\left(\mathbb{R}^{n}\right)$ possui. Por exemplo, se conhecemos a imersão $W^{m, p}\left(\mathbb{R}^{n}\right) \rightarrow L^{q}\left(\mathbb{R}^{n}\right)$, então podemos inferir a imersão $W^{m, p}(\Omega) \rightarrow L^{q}(\Omega)$ pelo cálculo seguinte:

$$
\|u\|_{0, q, \Omega} \leq\|E u\|_{0, p, \mathbb{R}^{n}} \leq K_{1}\|E u\|_{m, p, \mathbb{R}^{n}} \leq K_{1} K\|u\|_{m, p, \Omega}
$$

\section{B.2 O Teorema de Extensão de Calderón}

Definição B.4 Dado um ponto $x \in \mathbb{R}^{n}$, uma bola aberta $B_{1}$ com centro em $x$ e uma bola aberta $B_{2}$ que não contém ao ponto $x$, definimos o cone finito com vértice em $x$, como o cunjunto

$$
C_{x}=B_{1} \cap\left\{x+\lambda(y-x): y \in B_{2}, \lambda>0\right\} .
$$

Denotaremos por $x+C_{0}=\left\{x+y: y \in C_{0}\right\}$ o cone finito com vértice em $x$ obtido da translação paralela de um cone finito $C_{0}$ com vértice na origem.

Um cobrimento aberto $\mathcal{O}$ de um conjunto $S \subset \mathbb{R}^{n}$ é chamado localmente finito, se qualquer conjunto compacto de $\mathbb{R}^{n}$ intersecta no máximo um número finito de elementos de $\mathcal{O}$. 
Agora definimos uma propriedade de regularidade para um $\Omega$ aberto.

Definição B.5 Diremos que $\Omega$ possui a propriedade uniforme de cone, se existir um cobrimento aberto localmente finito $\left\{U_{j}\right\}$ da fronteira $\partial \Omega$, de $\Omega$, e uma correspondente seqüência de cones finitos $\left\{C_{j}\right\}$, cada um deles congruente com um cone finito $C$ fixo, tal que

1. para alguma constante $M$, qualquer $U_{j}$ tem diâmetro menor que $M$;

2. para algum $\delta>0, \bigcup_{j=1}^{\infty} U_{j} \supset \Omega_{\delta} \equiv\{x \in \Omega: \operatorname{dist}(x, \partial \Omega)<\delta\}$;

3. para qualquer $j, \bigcup_{x \in \Omega \cap U_{j}}\left(x+C_{j}\right) \equiv Q_{j} \subset \Omega$;

4. para alguma constante $R$, qualquer coleção de $R+1$ dos conjuntos $Q_{j}$ têm interseção vazia.

\section{Teorema B.6 (de Extensão de Calderón)}

Seja $\Omega$ um domínio em $\mathbb{R}^{n}$ que possui a propriedade uniforme de cone, modificada como segue:

1. o cobrimento $\left\{U_{j}\right\}$ da $\partial \Omega$ é finito, $e$

2. os conjuntos $U_{j}$ não necessariamente são limitados.

Então, para qualquer $m \in\{1,2, \ldots\}$ e qualquer $p, 1<p<\infty$, existe um $(m, p)$ operador de extensão simples $E$, para $\Omega$. 


\section{Desigualdades e Teoremas}

\section{Mencionados}

\section{C.1 Desigualdade de Hölder}

Sejam $1 \leq p, q, r \leq \infty$ tais que $p^{-1}+q^{-1}=r^{-1}$. Se $f \in L^{p}\left(\mathbb{R}^{n}\right)$ e $g \in L^{q}\left(\mathbb{R}^{n}\right)$, então $f g \in L^{r}\left(\mathbb{R}^{n}\right)$. Além disso

$$
\|f g\|_{r} \leq\|f\|_{p}\|g\|_{q} .
$$

\section{C.2 Desigualdade de Hardy-Littlewood-Sobolev}

Consideremos a seguinte família de operadores de integração fraccionária:

$$
I_{r} f(x)=\int_{\mathbb{R}^{n}}|x-y|^{n / r} f(y) d y .
$$

Se $r>1$ e

$$
\frac{1}{r}=1-\left(\frac{1}{p}-\frac{1}{q}\right)
$$

para alguns $1<p<q<\infty$, então

$$
\left\|I_{r} f\right\|_{L^{q}\left(\mathbb{R}^{n}\right)} \leq C_{p, q}\|f\|_{L^{p}\left(\mathbb{R}^{n}\right)}
$$


C.3 Teorema de Plancherel

\section{C.3 Teorema de Plancherel}

Se $u \in L^{2}\left(\mathbb{R}^{n}\right)$, então $\widehat{u} \in L^{2}\left(\mathbb{R}^{n}\right)$ e

$$
\|\widehat{u}\|_{2}^{2}=(2 \pi)^{n}\|u\|_{2}^{2} .
$$




\section{Referências Bibliográficas}

[1] J. Bergh e J. Löfström, Interpolation Spaces, Springer-Verlag, 1976.

[2] M. Gel'fand e G. E. Śilov, Generalized functions, Vol. I: Operations on them, Fizmatgiz, Moscow, 1958; English transl., Academic Press, New York, 1964.

[3] V. Georgiev, Weighted estimate for the wave equation, Nonlinear waves, Proceedings of the Fourth MSJ International Research Institute, vol. 1, Hokkaido Univ., 1996, pp. 71-80.

[4] V. Georgiev, H. Lindblad e C. D. Sogge, Weighted Strichartz estimates and global existence for semilinear wave equations, Amer. J. Math. 119 (1997), no. 6, 1291-1319.

[5] R. Glassey, Existence in the large for $\square u=f(u)$ in two dimensions, Math. Z. 178 (1981), 233-261.

[6] L. Hormander, Fourier integrals i, Acta Math 127 (1971), 79-183.

[7] Lars Hörmander, Estimatives for tranlation invariant operators in $l^{p}$ spaces, Acta. Math. 104 (1960), 93-140.

[8] _ Lectures in nonlinear hyperbolic differential equations, 117-130, Springer-Verlag, 1997, pp. 117-130.

[9] F. John, Blow-up of solutions of nonlinear wave equations in three space dimensions, Manuscrypta Math. 28 (1979), 235-265. 
[10] H. Kubo, On the critical decay and power for semilinear wave equations in odd space dimensions, Nonlinear Anal. 28 (1997), no. 2, 327-357.

[11] H. Lindblad e C. D. Sogge, On existence and scattering with minimal regularity for semilinear wave equations., J. Func. Anal. 130 (1995), 357-426.

[12] — L L L L L Amer. J. Math. 118 (1996), 1047-1135.

[13] W. Littman, The wave operator and $L_{p}$ norms, J. Math. Mech. 12 (1963), 55-68.

[14] J. Schaeffer, The equation $\square u=|u|^{p}$ for the critical value of p, Proc. Royal Soc. Edinburgh Sect. A 101 (1985), 31-44.

[15] T. Sideris, Nonexistence of global solutions to semilinear wave equations in high dimensions, Comm. Partial Differential Equations 12 (1987), 378-406.

[16] C. D. Sogge e E. M. Stein, Averages of functions over hypersurfaces: Smoothness of generalized Radon transforms, J. Anal. Math. 54 (1990), 165-188.

[17] Christopher D. Sogge, Fourier Integrals in Classical Analysis, Cambridge, 1993.

[18] Elias M. Stein, Interpolation of linear operators, Trans. Amer. Math. Soc. 83 (1956), 482-492.

[19] W. Strauss, Nonlinear scattering theory, Scattering Theory in Mathematical Physics, Reidel, Dordrect, 1979, pp. 53-79.

[20] _ Nonlinear scattering at low energy, J. Func. Anal. 41 (1981), 110-133.

[21] R. Strichartz, Convolutions with kernels having singularities on a sphere, Trans. Amer. Math. Soc. 148 (1970), 461-471.

[22] _ A priori estimates for the wave equation and some aplications, J. Func. Anal. 41 (1981), 110-133.

[23] Y. Zhou, Cauchy problem for semilinear wave equations with small data in four space dimensions, J. Differential Equations 8 (1995), 135-144. 